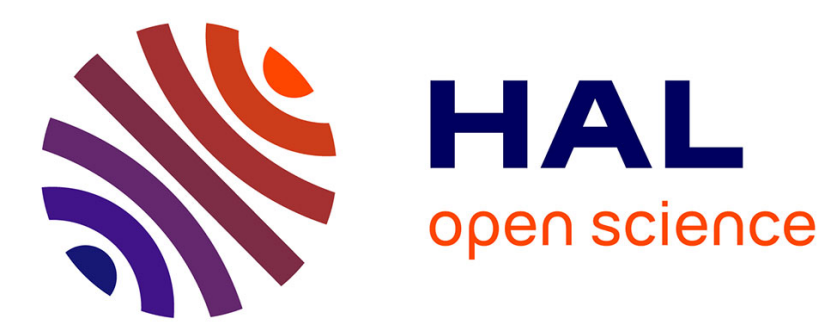

\title{
Existence and uniqueness of solution to the two-phase Stefan problem with convection
}

Viorel Barbu, Ioana Ciotir, Ionut Danaila

\section{To cite this version:}

Viorel Barbu, Ioana Ciotir, Ionut Danaila. Existence and uniqueness of solution to the two-phase Stefan problem with convection. 2020. hal-02615472

\section{HAL Id: hal-02615472 \\ https://hal.science/hal-02615472}

Preprint submitted on 22 May 2020

HAL is a multi-disciplinary open access archive for the deposit and dissemination of scientific research documents, whether they are published or not. The documents may come from teaching and research institutions in France or abroad, or from public or private research centers.
L'archive ouverte pluridisciplinaire HAL, est destinée au dépôt et à la diffusion de documents scientifiques de niveau recherche, publiés ou non, émanant des établissements d'enseignement et de recherche français ou étrangers, des laboratoires publics ou privés. 


\title{
Existence and uniqueness of solution to the two-phase Stefan problem with convection
}

\author{
Viorel BARBU ${ }^{a}$, Ioana $\mathrm{CIOTIR}^{b}$, Ionut DANAILA ${ }^{c}$ \\ ${ }^{a}$ Octav Mayer Institute of Mathematics of the Romanian Academy, Iasi, Romania \\ ${ }^{b}$ Normandie University, INSA de Rouen Normandie, \\ LMI (EA 3226 - FR CNRS 3335), 76000 Rouen, France \\ 685 Avenue de l'Université, 76801 St Etienne du Rouvray cedex \\ ${ }^{c}$ Laboratoire de Mathématiques Raphaël Salem \\ UMR 6085 CNRS-Université de Rouen Normandie \\ Avenue de l'Université, BP.12F76801 Saint-Étienne-du-Rouvray
}

May 19, 2020

\begin{abstract}
The well posedness of the two-phase Stefan problem with convection is established in $L^{1}$. First we consider the case with a singular enthalpy and we fix the convection velocity. In the second part of the paper we study the case of a smoothed enthalpy, but the convection velocity is the solution to a Navier-Stokes equation. In the last section we give some numerical illustrations of a physical case simulated using the models studied in the paper.
\end{abstract}

Key word: Stefan problem, Navier-Stokes equation, convection velocity, monotone operators

AMS [2020] 35D99, 35Q30, 35R35, 35Q79

\section{Introduction}

In this work we are interested in the study of a solid-liquid phase transition problem with convection. More precisely, we prove existence and uniqueness of the solution for this problem in two different settings: the case with degenerated enthalpy function and a fixed vectorial velocity function in the convection term and the case with a non-degenerated enthalpy function and a convection term modelled by a Navier-Stokes equation.

Our approach is based on the interpretation of the Stefan problem as a porous media equation with non-linear transport (drift) term.

We start by considering the following free boundary problem with convection on an open bounded subset $\mathcal{O}$ of $\mathbb{R}^{d}$ with smooth boundary $\partial \mathcal{O}$ 


$$
\left\{\begin{array}{l}
C_{1} \frac{\partial \theta}{\partial t}-\operatorname{div}\left(k_{1} \nabla_{\xi} \theta\right)+\operatorname{div}(Y \eta(\theta))=F, \quad \text { if } \theta<0, \\
C_{2} \frac{\partial \theta}{\partial t}-\operatorname{div}\left(k_{2} \nabla_{\xi} \theta\right)+\operatorname{div}(Y \eta(\theta))=F, \quad \text { if } \theta>0, \\
\left(k_{2} \nabla \theta^{+}-k_{1} \nabla \theta^{-}\right) \cdot N_{\xi}^{-}=l N_{t}, \quad \text { on the interface } \theta=0, \\
\theta^{+}=\theta^{-}=0, \quad \text { on the interface } \theta=0, \\
\theta(t, \xi)=0, \quad \text { on } \partial \mathcal{O} \times(0, T), \\
\theta(0, \xi)=\theta_{0}(\xi), \quad \text { on } \mathcal{O},
\end{array}\right.
$$

which models the melting process of a solid (for example ice) in the presence of a heating source $F$. Here $\theta^{+}$and $\theta^{-}$are the right and left limits of the free boundary situated between the solid and the liquid phase, respectively, and $N=N(\xi, t)$ is the unit normal to the interface.

We denote by $k_{1}$ and $k_{2}$ the thermal conductivity of the solid and liquid phases. Similarly, $C_{1}$ and $C_{2}$ are the specific heat for the two phases.

The function $\eta: \mathbb{R} \rightarrow \mathbb{R}$ is assumed to be a Lipschitz continuous, nondecreasing function which vanish in the solid phase and such that $\eta(0)=0$. The physical interpretation of this term is that only the liquid phase is allowed to move.

The vectorial function $Y$ describes the velocity field and is assumed to be $Y \in\left(L^{\infty}(\mathcal{O})\right)^{d}$ and fixed in the degenerated case, and derived as a solution to a Navier-Stokes equation in the non-degenerated case. Finally $l$ is assumed to be the latent heat.

In the literature there exists a large number of references concerning the Stefan problem. Let us mention [13], [14], [21] and [23] for the physical motivation and the mathematical treatment of the problem. The case of a heterogeneous media without convection was studied in [20].

Concerning the Stefan problem with convection we refer to [8] for the stationary case, [7] for the study of renormalized solutions and [1], [15] for a numerical point of view.

A classical approach (see e.g. [2], [14]) as above problem is to write it as a non-linear multi-valued problem of monotone type. Namely, we can rewrite (1.1) as

$$
\begin{cases}\frac{\partial}{\partial t} \gamma(\theta)-\operatorname{div}\left(k(\theta) \nabla_{\xi} \theta\right)+\operatorname{div}(Y \eta(\theta))=F, & \text { in }(0, T) \times \mathcal{O} \\ \theta(0, \xi)=\theta_{0}(\xi), & \text { in } \mathcal{O}, \\ \theta(t, \xi)=0, & \text { on } \partial \mathcal{O},\end{cases}
$$

where $\gamma$ is the enthalpy function given by

$$
\gamma(r)=C(r)+l H(r)
$$

where

$$
C(r)= \begin{cases}C_{1} r, & r \leq 0 \\ C_{2} r, & r>0\end{cases}
$$


and $H$ is the Heaviside function

$$
H(r)= \begin{cases}0, & r<0 \\ {[0,1],} & r=0 \\ 1, & r>0\end{cases}
$$

whilst

$$
k(r)= \begin{cases}k_{1}, & r \leq 0, \\ k_{2}, & r>0 .\end{cases}
$$

Let us denote by $K$ the primitive of $k$ and rewrite (1.2) as

$$
\begin{cases}\frac{\partial}{\partial t} \gamma(\theta)-\Delta K(\theta)+\operatorname{div}(Y \eta(\theta))=F, & \text { in }(0, T) \times \mathcal{O} \\ \theta(0, \xi)=\theta_{0}(\xi), & \text { in } \mathcal{O}, \\ \theta(t, \xi)=0, & \text { on } \partial \mathcal{O} .\end{cases}
$$

By the change of variable $\gamma(\theta)=X$ we can write the latter as

$$
\begin{cases}\frac{\partial X}{\partial t}-\Delta \Psi(X)+\operatorname{div}\left(Y \eta\left(\gamma^{-1}(X)\right)\right)=F, & \text { in }(0, T) \times \mathcal{O}, \\ X(0, \xi)=X_{0}(\xi), & \text { in } \mathcal{O}, \\ X(t, \xi)=0, & \text { on } \partial \mathcal{O},\end{cases}
$$

where

$$
\Psi(r)= \begin{cases}k_{1} C_{1}^{-1} r, & r<0, \\ 0, & r \in[0, l] \\ k_{2} C_{2}^{-1}(r-l), & r>l,\end{cases}
$$

and

$$
\gamma^{-1}(r)= \begin{cases}C_{1}^{-1} r, & r<0, \\ 0, & r \in[0, l] \\ C_{2}^{-1}(r-l), & r>l .\end{cases}
$$

Here we shall study a more general equation of the form

$$
\begin{cases}\frac{\partial X}{\partial t}-\Delta \beta(X)+\operatorname{div}(Y \alpha(X))=F, & \text { in }(0, T) \times \mathcal{O} \\ X(0)=X_{0}, & \text { in } \mathcal{O} \\ \beta(X)=0, & \text { on }(0, T) \times \partial \mathcal{O}\end{cases}
$$

where $Y \in\left(L^{\infty}(\mathcal{O})\right)^{d}$ and $\alpha, \beta: \mathbb{R} \rightarrow \mathbb{R}$ are assumed to satisfy the following hypotheses

i) $\beta$ and $\alpha$ are continuous, monotonically nondecreasing functions satisfying

$$
\begin{gathered}
\beta(0)=\alpha(0)=0, \quad|\alpha(r)|+|\beta(r)| \leq C_{3}|r|, \quad \forall r \in \mathbb{R} \\
r \beta(r) \geq C_{4} r^{2}+C_{5}, \quad \forall r \in \mathbb{R} \\
|\alpha(r)-\alpha(s)| \leq C_{6}|\beta(r)-\beta(s)|, \quad \forall r, s \in \mathbb{R}
\end{gathered}
$$

for some positive constants $C_{i}, i=3,4,6$ and $C_{5} \in \mathbb{R}$. 
Equation (1.7) reduces to (1.4) for $\beta=\Psi, \alpha=\eta \circ \gamma^{-1}$ and the assumptions above hold if $\eta$ is Lipschitz and monotonically nondecreasing such that $\eta(0)=0$.

In the following we shall denote by $C$ several positive constants, that may change in the chains of estimates.

The structure of the paper is the following. In Section 2 we prove the existence and uniqueness to the equation (1.7) and in particular to equation (1.4) with a singular enthalpy and fixed convection velocity in the space $L^{1}(\mathcal{O})$. In Section 3 we study the case of a smoothed enthalpy, but with the convection velocity as solution to a Navier-Stokes equation, by using a fixed point argument. In Section 4 we give some numerical illustration.

To the best of our knowledge the problem from Section 2 was never treated in this framework and the one from Section 3 was never treated at all. The numerical results illustrate the interest of this theoretical formulation of the problem.

\section{Notations}

Denote by $L^{p}(\mathcal{O}), 1 \leq p<\infty$, the space of Lebesgue $p$-integrable functions on $\mathcal{O}$ with the norm denoted $|\cdot|_{p}$, the scalar product in $L^{2}(\mathcal{O})$ is denoted $(., .)_{2}$ and by $W^{1, p}(\mathcal{O})=\left\{u \in L^{p}(\mathcal{O}) ; D_{j} u \in L^{p}(\mathcal{O}), j=1,2, \ldots, d\right\}$ and $W_{0}^{1, p}(\mathcal{O})=$ $\left\{u \in W^{1, p}(\mathcal{O}), u=0\right.$ on $\left.\partial \mathcal{O}\right\}$ the standard Sobolev spaces on $\mathcal{O} . H^{-1}(\mathcal{O})$ is the dual of Sobolev space $H_{0}^{1}(\mathcal{O})$ with the norm $|u|_{-1}=((-\Delta) u, u)_{2}^{1 / 2}$ where $-\Delta$ is the Laplace operator with homogeneous Dirichlet conditions. Denote by $\operatorname{Lip}(\mathbb{R})$ the space of Lipschitz functions on $\mathbb{R}$ with the norm $\|\cdot\|_{\text {Lip }}$.

Let $X$ be a Banach space. The operator $A: X \rightarrow X$ (eventually multivalued) is said to be accretive if

$$
\left|u_{1}-u_{2}\right|_{X} \leq\left|u_{1}-u_{2}+\lambda\left(v_{1}-v_{2}\right)\right|_{X}, \quad \forall \lambda>0,
$$

where $v_{i} \in A u_{i}, i=1,2, u_{i} \in D(A)$.

The operator $A$ is said to be $m$-accretive if it is accretive and $R(I+\lambda A)=$ $X, \forall \lambda>0$ (equivalently for same $\lambda>0$ ). (See e.g. [2]). Here $D(A)=$ $\{u \in X ; A u \neq \emptyset\}$ is the domain of $A$ and $R(I+\lambda A)$ is the range of $I+\lambda A$. In particular it follows that if $A$ is $m$-accretive then

$$
\left|(I+\lambda A)^{-1} u_{1}-(I+\lambda A)^{-1} u_{2}\right|_{X} \leq\left|u_{1}-u_{2}\right|_{X}, \quad \forall u_{1}, u_{2} \in X, \lambda>0 .
$$

As a matter of fact, $A$ is $m$-accretive, if and only if $R(I+\lambda A)=X, \forall \lambda>0$ and (1.12) holds. 


\section{Phase transition with degenerated enthalpy and fixed convection velocity}

We study here the existence and uniqueness of the solution to the equation

$$
\begin{cases}\frac{\partial X}{\partial t}-\Delta \beta(X)+\operatorname{div}(Y \alpha(X))=F, & \text { in }(0, T) \times \mathcal{O}, \\ X(0, \xi)=X_{0}(\xi), & \text { in } \mathcal{O}, \\ \beta(X(t, \xi))=0, & \text { on }(0, T) \times \partial \mathcal{O}\end{cases}
$$

We assume that hypothesis $i$ ) hold and also that $Y$ is time independent and

$$
Y \in\left(L^{\infty}(\mathcal{O})\right)^{d}, \operatorname{div} Y \in L^{\infty}(\mathcal{O}) .
$$

We are going to represent (2.1) as an infinite dimensional Cauchy problem of the form

$$
\left\{\begin{array}{l}
\frac{d X}{d t}+A X=F, \quad t \in[0, T] \\
X(0)=X_{0}
\end{array}\right.
$$

where $A$ is an $m$-accretive operator in $L^{1}(\mathcal{O})$.

To this purpose we define the operator $A_{0}: L^{1}(\mathcal{O}) \rightarrow L^{1}(\mathcal{O})$

$$
A_{0}(u)=-\Delta \beta(u)+\operatorname{div}(Y \alpha(u)), \quad \forall u \in \widehat{D},
$$

where

$$
\widehat{D}=\left\{u \in L^{1}(\mathcal{O}) ;-\Delta \beta(u)+\operatorname{div}(Y \alpha(u)) \in L^{1}(\mathcal{O})\right\}
$$

Here $\Delta$ and div are taken in the sense of Schwartz distributions, that is the space $\mathcal{D}^{\prime}(\mathcal{O})$.

Theorem 1 For each $f \in L^{1}(\mathcal{O})$ and $\lambda>0$ there is a solution $u(\lambda, f) \in \widehat{D}$ to equation

$$
u+\lambda A_{0} u=f
$$

Moreover, we have

$$
u\left(\lambda_{2}, f\right)=u\left(\lambda_{1}, \frac{\lambda_{1}}{\lambda_{2}} f+\left(1-\frac{\lambda_{1}}{\lambda_{2}}\right) u\left(\lambda_{2}, f\right)\right), \forall 0<\lambda_{1} \leq \lambda_{2}, \quad f \in L^{1}(\mathcal{O}),
$$

and

$$
\left|u\left(\lambda, f_{1}\right)-u\left(\lambda, f_{2}\right)\right|_{1} \leq\left|f_{1}-f_{2}\right|_{1}, \forall \lambda>0, f_{1}, f_{2} \in L^{1}(\mathcal{O}) .
$$

Furtheron, if $f \geq 0$ a.e. in $\mathcal{O}$ then

$$
u(\lambda, f) \geq 0 \text { a.e. in } \mathcal{O} \text {. }
$$

Assume further that $\alpha \in L^{\infty}(\mathbb{R})$. Then

$$
\begin{gathered}
u(\lambda, f) \in W_{0}^{1, q}(\mathcal{O}) \text { for } q \in\left(1, \frac{d}{d-1}\right) \\
|u(\lambda, f)|_{W_{0}^{1, q}(\mathcal{O})} \leq C|f|_{1}, \quad \forall f \in L^{1}(\mathcal{O}), \lambda>0 .
\end{gathered}
$$


It should be noted that Theorem 1 does not imply the $m$-accretivity of operator $A_{0}$ with domain $\widehat{D}$ because, in general, $A_{0}$ is not accretive on $\widehat{D} \subset$ $L^{1}(\mathcal{O})$. The exact meaning of Theorem 1 is that, for each $\lambda>0$ and $f \in L^{1}(\mathcal{O})$, there is one solution to equation (2.4) (perhaps not unique) which satisfies the contraction (2.6). As a matter of fact $u(\lambda, f)$ is a single valued section of the multivalued operator

$$
f \rightarrow\left(I+\lambda A_{0}\right)^{-1} f, \quad \forall f \in L^{1}(\mathcal{O}),
$$

that is

$$
u(\lambda, f) \in\left(I+\lambda A_{0}\right)^{-1} f, \quad \forall f \in L^{1}(\mathcal{O}), \lambda>0 .
$$

Note also that in the case of equation (1.4) condition $\alpha \in L^{\infty}(\mathbb{R})$ reduces to $\eta \in \operatorname{Lip}(\mathbb{R}) \cap L^{\infty}(\mathbb{R})$.

Proof of Theorem 1. We define, for each $\varepsilon>0$ fixed, the operator

$$
A_{\varepsilon}(u)=-\Delta(\beta(u)+\varepsilon u)+\operatorname{div}(Y \alpha(u))
$$

with

$$
D\left(A_{\varepsilon}\right)=\left\{u \in H_{0}^{1}(\mathcal{O}) ;-\Delta(\beta(u)+\varepsilon u)+\operatorname{div}(Y \alpha(u)) \in L^{1}(\mathcal{O})\right\} .
$$

Let us start with the proof of the fact that the range of $I+\lambda A_{\varepsilon}$ is all of $L^{2}(\mathcal{O})$. To this end, we fix $f \in L^{2}(\mathcal{O})$ and consider the equation

$$
u+\lambda A_{\varepsilon}(u)=f,
$$

that is

$$
\begin{cases}u-\lambda \Delta(\beta(u)+\varepsilon u)+\lambda \operatorname{div}(Y \alpha(u))=f, & \text { in } \mathcal{D}^{\prime}(\mathcal{O}), \\ u=0, & \text { on } \partial \mathcal{O} .\end{cases}
$$

Equivalently,

$$
(-\Delta)^{-1} u+\lambda \beta(u)+\lambda \varepsilon u+\lambda(-\Delta)^{-1} \operatorname{div}(Y \alpha(u))=(-\Delta)^{-1} f .
$$

Here $-\Delta$ is the Laplace operator with homogeneous boundary conditions.

We consider the operator $K: L^{2}(\mathcal{O}) \rightarrow L^{2}(\mathcal{O})$ defined by

$$
K(u)=\lambda \beta(u)
$$

and $H_{\varepsilon}: L^{2}(\mathcal{O}) \rightarrow L^{2}(\mathcal{O})$ defined by

$$
H_{\varepsilon}(u)=(-\Delta)^{-1} u+\lambda \varepsilon u+\lambda(-\Delta)^{-1} \operatorname{div}(Y \alpha(u)) .
$$

We see that $K$ is $m$-accretive in $L^{2}(\mathcal{O})$ and, since $|\beta(r)| \leq C|r|$ for $\forall r \in \mathbb{R}$, $K$ is also continuous in $L^{2}(\mathcal{O})$.

Concerning $H_{\varepsilon}$, taking into account that

$$
|\operatorname{div}(Y(\alpha(u)-\alpha(v)))|_{-1} \leq|Y(\alpha(u)-\alpha(v))|_{2},
$$


we obtain

$$
\begin{aligned}
& \left\langle H_{\varepsilon}(u)-H_{\varepsilon}(v), u-v\right\rangle_{2} \\
= & |u-v|_{-1}^{2}+\lambda \varepsilon|u-v|_{2}^{2}+\lambda\langle\operatorname{div}(Y(\alpha(u)-\alpha(v))), u-v\rangle_{-1} \\
\geq & |u-v|_{-1}^{2}+\lambda \varepsilon|u-v|_{2}^{2}-\frac{\lambda^{2}}{2}|Y|_{\infty}^{2} L^{2}|u-v|_{2}^{2}-\frac{1}{2}|u-v|_{-1}^{2} \\
\geq & \lambda\left(\varepsilon-\frac{\lambda}{2}|Y|_{\infty}^{2} L^{2}\right)|u-v|_{2}^{2}
\end{aligned}
$$

where $L=\|\alpha\|_{\text {Lip }}$.

For $\lambda$ sufficiently small, i.e.,

$$
\frac{\lambda}{2}|Y|_{\infty}^{2} L^{2}<\frac{\varepsilon}{2} \Leftrightarrow \lambda<\lambda_{\varepsilon}=\frac{\varepsilon}{2|Y|_{\infty}^{2} L^{2}}
$$

we obtain that

$$
\left\langle H_{\varepsilon}(u)-H_{\varepsilon}(v), u-v\right\rangle_{2} \geq \frac{\lambda \varepsilon}{2}|u-v|_{2}^{2} .
$$

Then, for $\lambda<\lambda_{\varepsilon}$, where $\lambda_{\varepsilon}$ is sufficiently small, $H_{\varepsilon}$ is accretive, continuous and coercive on $L^{2}(\mathcal{O})$.

This implies that (see e.g. [2], page 37) $K+H_{\varepsilon}$ is $m$-accretive, continuous and coercive and therefore surjective.

Hence, for each $f \in L^{2}(\mathcal{O})$ and $\lambda<\lambda_{\varepsilon}$, equation (2.11) has a unique solution $u_{\varepsilon}=u_{\varepsilon}(\lambda, f) \in L^{2}(\mathcal{O})$, and therefore we have $\beta_{\varepsilon}\left(u_{\varepsilon}\right)=\beta\left(u_{\varepsilon}\right)+\varepsilon u_{\varepsilon} \in H_{0}^{1}(\mathcal{O})$ and, by the form of $\beta$, we have also that $u_{\varepsilon} \in H_{0}^{1}(\mathcal{O})$ and $\beta\left(u_{\varepsilon}\right) \in H_{0}^{1}(\mathcal{O})$.

If we denote by $u_{\varepsilon}^{i}$ the solution to $u_{\varepsilon}^{i}+\lambda A_{\varepsilon}\left(u_{\varepsilon}^{i}\right)=f_{i}$, for each $f_{i} \in L^{2}(\mathcal{O}) \subset$ $L^{1}(\mathcal{O})$ and for each $i \in\{1,2\}$, we have also that

$$
\left|u_{\varepsilon}^{1}-u_{\varepsilon}^{2}\right|_{1} \leq\left|f_{1}-f_{2}\right|_{1}, \forall f_{1}, f_{2} \in L^{2}(\mathcal{O}) .
$$

In order to prove the later estimate, we take the following Lipschitz approximation of the sign multivalued function, $\chi_{\delta}: \mathbb{R} \rightarrow \mathbb{R}$,

$$
\chi_{\delta}(r)= \begin{cases}1, & r \geq \delta, \\ \frac{r}{\delta}, & |r|<\delta, \\ -1, & r<-\delta,\end{cases}
$$

and we use a similar approach to the one used in [6].

Namely, we multiply the equation

$$
\begin{gathered}
u_{\varepsilon}^{1}-u_{\varepsilon}^{2}-\lambda \Delta\left(\beta_{\varepsilon}\left(u_{\varepsilon}^{1}\right)-\beta_{\varepsilon}\left(u_{\varepsilon}^{2}\right)\right) \\
+\lambda \operatorname{div}\left(Y\left(\alpha\left(u_{\varepsilon}^{1}\right)-\alpha\left(u_{\varepsilon}^{2}\right)\right)\right)=f_{1}-f_{2},
\end{gathered}
$$

where $\beta_{\varepsilon}(r)=\beta(r)+\varepsilon r$, by $\chi_{\delta}\left(\beta_{\varepsilon}\left(u_{\varepsilon}^{1}\right)-\beta_{\varepsilon}\left(u_{\varepsilon}^{2}\right)\right)$ and integrate over $\mathcal{O}$. 
We get

$$
\begin{aligned}
& \int_{\mathcal{O}}\left(u_{\varepsilon}^{1}-u_{\varepsilon}^{2}\right) \chi_{\delta}\left(\beta_{\varepsilon}\left(u_{\varepsilon}^{1}\right)-\beta_{\varepsilon}\left(u_{\varepsilon}^{2}\right)\right) d \xi \\
& +\int_{\mathcal{O}} \nabla\left(\beta_{\varepsilon}\left(u_{\varepsilon}^{1}\right)-\beta_{\varepsilon}\left(u_{\varepsilon}^{2}\right)\right) \nabla \chi_{\delta}\left(\beta_{\varepsilon}\left(u_{\varepsilon}^{1}\right)-\beta_{\varepsilon}\left(u_{\varepsilon}^{2}\right)\right) d \xi \\
& +\lambda \int_{\mathcal{O}} \operatorname{div}\left(Y\left(\alpha\left(u_{\varepsilon}^{1}\right)-\alpha\left(u_{\varepsilon}^{2}\right)\right)\right) \chi_{\delta}\left(\beta_{\varepsilon}\left(u_{\varepsilon}^{1}\right)-\beta_{\varepsilon}\left(u_{\varepsilon}^{2}\right)\right) d \xi \\
\leq & \int_{\mathcal{O}}\left(f_{1}-f_{2}\right) \chi_{\delta}\left(\beta_{\varepsilon}\left(u_{\varepsilon}^{1}\right)-\beta_{\varepsilon}\left(u_{\varepsilon}^{2}\right)\right) d \xi .
\end{aligned}
$$

We calculate the term

$$
\begin{aligned}
& I_{\delta}= \int_{\mathcal{O}} \operatorname{div}\left(Y(\xi)\left(\alpha\left(u_{\varepsilon}^{1}\right)-\alpha\left(u_{\varepsilon}^{2}\right)\right)\right) \chi_{\delta}\left(\beta_{\varepsilon}\left(u_{\varepsilon}^{1}\right)-\beta_{\varepsilon}\left(u_{\varepsilon}^{2}\right)\right) d \xi \\
&=-\int_{\mathcal{O}}\left(Y(\xi)\left(\alpha\left(u_{\varepsilon}^{1}\right)-\alpha\left(u_{\varepsilon}^{2}\right)\right)\right) \chi_{\delta}^{\prime}\left(\beta_{\varepsilon}\left(u_{\varepsilon}^{1}\right)-\beta_{\varepsilon}\left(u_{\varepsilon}^{2}\right)\right) \\
& \cdot \nabla\left(\beta_{\varepsilon}\left(u_{\varepsilon}^{1}\right)-\beta_{\varepsilon}\left(u_{\varepsilon}^{2}\right)\right) d \xi \\
&=-\frac{1}{\delta} \int_{\left[\left|\beta_{\varepsilon}\left(u_{\varepsilon}^{1}\right)-\beta_{\varepsilon}\left(u_{\varepsilon}^{2}\right)\right| \leq \delta\right]}\left(Y(\xi)\left(\alpha\left(u_{\varepsilon}^{1}\right)-\alpha\left(u_{\varepsilon}^{2}\right)\right)\right) \\
& \cdot \nabla\left(\beta_{\varepsilon}\left(u_{\varepsilon}^{1}\right)-\beta_{\varepsilon}\left(u_{\varepsilon}^{2}\right)\right) d \xi .
\end{aligned}
$$

We note that

$$
\begin{aligned}
I_{\delta} & \leq\left|I_{\delta}\right| \\
& \leq \frac{|Y|_{\infty} L}{\delta} \int_{\left[\left|\beta_{\varepsilon}\left(u_{\varepsilon}^{1}\right)-\beta_{\varepsilon}\left(u_{\varepsilon}^{2}\right)\right| \leq \delta\right]}\left|u_{\varepsilon}^{1}-u_{\varepsilon}^{2}\right|\left|\nabla\left(\beta_{\varepsilon}\left(u_{\varepsilon}^{1}\right)-\beta_{\varepsilon}\left(u_{\varepsilon}^{2}\right)\right)\right|_{\mathbb{R}^{d}} d \xi .
\end{aligned}
$$

Keeping in mind that

$$
\left(\beta_{\varepsilon}(u)-\beta_{\varepsilon}(v)\right)(u-v) \geq \varepsilon|u-v|^{2}, \forall u, v \in \mathbb{R},
$$

we get that

$$
|u-v| \leq \frac{1}{\varepsilon}\left|\beta_{\varepsilon}(u)-\beta_{\varepsilon}(v)\right|
$$

and using this relation in the previous computation we obtain that

$$
\begin{aligned}
\left|I_{\delta}\right| & \leq \frac{|Y|_{\infty} L}{\delta \varepsilon} \int_{\left[\left|\beta_{\varepsilon}\left(u_{\varepsilon}^{1}\right)-\beta_{\varepsilon}\left(u_{\varepsilon}^{2}\right)\right| \leq \delta\right]}\left|\beta_{\varepsilon}(u)-\beta_{\varepsilon}(v)\right| \\
& \leq \frac{|Y|_{\infty} L}{\varepsilon} \int_{\left[\left|\beta_{\varepsilon}\left(u_{\varepsilon}^{1}\right)-\beta_{\varepsilon}\left(u_{\varepsilon}^{2}\right)\right| \leq \delta\right]}\left|\nabla\left(\beta_{\varepsilon}\left(u_{\varepsilon}^{1}\right)-\beta_{\varepsilon}\left(u_{\varepsilon}^{2}\right)\right)\right|_{\mathbb{R}^{d}} d \xi, \forall \varepsilon>0 .
\end{aligned}
$$

Since $\nabla\left(\beta_{\varepsilon}\left(u_{\varepsilon}^{1}\right)-\beta_{\varepsilon}\left(u_{\varepsilon}^{2}\right)\right)=0$ a.e. on $\left[\left|\beta_{\varepsilon}\left(u_{\varepsilon}^{1}\right)-\beta_{\varepsilon}\left(u_{\varepsilon}^{2}\right)\right|=0\right]$ we get that

$$
\lim _{\delta \rightarrow 0} \int_{\left[\left|\beta_{\varepsilon}\left(u_{\varepsilon}^{1}\right)-\beta_{\varepsilon}\left(u_{\varepsilon}^{2}\right)\right| \leq \delta\right]}\left|\nabla\left(\beta_{\varepsilon}\left(u_{\varepsilon}^{1}\right)-\beta_{\varepsilon}\left(u_{\varepsilon}^{2}\right)\right)\right|_{\mathbb{R}^{d}} d \xi=0
$$


and therefore $\lim _{\delta \rightarrow 0}\left|I_{\delta}\right|=0$.

We obtain that

$$
\lim _{\delta \rightarrow 0} I_{\delta}=0 .
$$

Going back to (2.12) and keeping in mind that

$$
\left|\chi_{\delta}\left(\beta_{\varepsilon}\left(u_{\varepsilon}^{1}\right)-\beta_{\varepsilon}\left(u_{\varepsilon}^{2}\right)\right)\right| \leq 1
$$

we have that

$$
\begin{aligned}
& \int_{\mathcal{O}}\left(u_{\varepsilon}^{1}-u_{\varepsilon}^{2}\right) \chi_{\delta}\left(\beta_{\varepsilon}\left(u_{\varepsilon}^{1}\right)-\beta_{\varepsilon}\left(u_{\varepsilon}^{2}\right)\right) d \xi+\lambda I_{\delta} \\
\leq & \int_{\mathcal{O}}\left|f_{1}-f_{2}\right| d \xi
\end{aligned}
$$

Since we have

$$
\left(u_{\varepsilon}^{1}-u_{\varepsilon}^{2}\right) \chi_{\delta}\left(\beta_{\varepsilon}\left(u_{\varepsilon}^{1}\right)-\beta_{\varepsilon}\left(u_{\varepsilon}^{2}\right)\right) \geq 0
$$

and $\chi_{\delta} \rightarrow \operatorname{sign}$ as $\delta \rightarrow 0$, we get by the Fatou's lemma that

$$
\int_{\mathcal{O}}\left(u_{\varepsilon}^{1}-u_{\varepsilon}^{2}\right) \operatorname{sign}\left(\beta_{\varepsilon}\left(u_{\varepsilon}^{1}\right)-\beta_{\varepsilon}\left(u_{\varepsilon}^{2}\right)\right) d \xi \leq \int_{\mathcal{O}}\left|f_{1}-f_{2}\right| d \xi
$$

which leads to

$$
\int_{\mathcal{O}}\left|u_{\varepsilon}^{1}-u_{\varepsilon}^{2}\right| d \xi \leq \int_{\mathcal{O}}\left|f_{1}-f_{2}\right| d \xi
$$

because, by the monotonicity of $\beta_{\varepsilon}$, we have

$$
\operatorname{sign}\left(\beta_{\varepsilon}\left(u_{\varepsilon}^{1}\right)-\beta_{\varepsilon}\left(u_{\varepsilon}^{2}\right)\right)=\operatorname{sign}\left(u_{\varepsilon}^{1}-u_{\varepsilon}^{2}\right) .
$$

We have shown therefore that $L^{2}(\mathcal{O}) \subset R\left(I+\lambda A_{\varepsilon}\right) \forall \lambda>0$ and that

$$
\left|\left(I+\lambda A_{\varepsilon}\right)^{-1} f_{1}-\left(I+\lambda A_{\varepsilon}\right)^{-1} f_{2}\right|_{1} \leq\left|f_{1}-f_{2}\right|_{1}, \forall f_{1}, f_{2} \in L^{2}(\mathcal{O}) \text {. }
$$

It follows also that $A_{\varepsilon}$ is accretive in $L^{1}(\mathcal{O})$. Indeed, we have

$$
\begin{aligned}
& \int_{\mathcal{O}}\left(A_{\varepsilon}(u)-A_{\varepsilon}(v)\right) \chi_{\delta}\left(\beta_{\varepsilon}(u)-\beta_{\varepsilon}(v)\right) d \xi \\
= & -\int_{\mathcal{O}} \Delta\left(\beta_{\varepsilon}(u)-\beta_{\varepsilon}(v)\right) \chi_{\delta}\left(\beta_{\varepsilon}(u)-\beta_{\varepsilon}(v)\right) d \xi \\
& +\int_{\mathcal{O}} \operatorname{div}(Y(\xi)(\alpha(u)-\alpha(v))) \chi_{\delta}\left(\beta_{\varepsilon}(u)-\beta_{\varepsilon}(v)\right) d \xi \\
\geq & \int_{\mathcal{O}}\left|\nabla\left(\beta_{\varepsilon}(u)-\beta_{\varepsilon}(v)\right)\right|_{\mathbb{R}^{d}}^{2} \chi_{\delta}^{\prime}\left(\beta_{\varepsilon}(u)-\beta_{\varepsilon}(v)\right) d \xi \\
& -|Y|_{\infty} L \int_{\mathcal{O}}|u-v| \chi_{\delta}^{\prime}\left(\beta_{\varepsilon}(u)-\beta_{\varepsilon}(v)\right)\left|\nabla\left(\beta_{\varepsilon}(u)-\beta_{\varepsilon}(v)\right)\right|_{\mathbb{R}^{d}} d \xi .
\end{aligned}
$$


By arguing as for $I_{\delta}$ above, we can see that the last integral converges to zero for $\delta \rightarrow 0$ and then

$$
\int_{\mathcal{O}}\left(A_{\varepsilon}(u)-A_{\varepsilon}(v)\right) \operatorname{sign}\left(\beta_{\varepsilon}(u)-\beta_{\varepsilon}(v)\right) d \xi \geq 0
$$

which implies directly that $A_{\varepsilon}$ is accretive in $L^{1}(\mathcal{O})$ due to the monotonicity of $\beta_{\varepsilon}$ (i.e., $\operatorname{sign}\left(\beta_{\varepsilon}(u)-\beta_{\varepsilon}(v)\right)=\operatorname{sign}(u-v)$ a.e. in $\mathcal{O}$ ).

In order to conclude de proof of Theorem 1 we should passe to the limit $u_{\varepsilon}=\left(I+\lambda A_{\varepsilon}\right)^{-1} f$, for $\varepsilon \rightarrow 0$.

To this end we come back to the equation

$$
u_{\varepsilon}-\lambda \Delta\left(\beta\left(u_{\varepsilon}\right)+\varepsilon u_{\varepsilon}\right)+\lambda \operatorname{div}\left(Y(\xi) \alpha\left(u_{\varepsilon}\right)\right)=f
$$

for $f \in L^{2}(\mathcal{O})$.

If $u_{\varepsilon}=u_{\varepsilon}(\lambda, f)$ it is easily seen by (2.10)-(2.11) that

$$
u_{\varepsilon}\left(\lambda_{2}, f\right)=u_{\varepsilon}\left(\lambda_{1}, \frac{\lambda_{1}}{\lambda_{2}} f+\left(1-\frac{\lambda_{1}}{\lambda_{2}}\right) u_{\varepsilon}\left(\lambda_{2}, f\right)\right), \forall 0<\lambda_{1} \leq \lambda_{2} .
$$

We note also that

$$
u_{\varepsilon}(\lambda, f) \geq 0 \text { a.e. in } \mathcal{O}, \forall \varepsilon>0, \forall \lambda>0
$$

if $f \geq 0$ a.e. in $\mathcal{O}$.

Here is the argument. If we multiply (2.15) by $u_{\varepsilon}^{-}$and integrate on $\mathcal{O}$ and taking into account that $\operatorname{sign} \beta(u)=\operatorname{sign} u$, we get

$$
\begin{aligned}
-\left|u_{\varepsilon}^{-}\right|_{2}^{2}-\lambda \int_{\mathcal{O}} \nabla \beta\left(u_{\varepsilon}^{-}\right) \cdot \nabla u_{\varepsilon}^{-} d \xi & =\lambda \int_{\mathcal{O}} Y \alpha\left(u_{\varepsilon}^{-}\right) \cdot \nabla u_{\varepsilon}^{-} d \xi+\int_{\mathcal{O}} f u_{\varepsilon}^{-} d \xi \\
& \geq \lambda \int_{\mathcal{O}} Y \cdot \nabla h\left(u_{\varepsilon}^{-}\right) d \xi \\
& =-\lambda \int_{\mathcal{O}} h\left(u_{\varepsilon}^{-}\right) \operatorname{div} Y d \xi
\end{aligned}
$$

where $h(r)=\int_{0}^{r} \alpha(s) d s$. This implies $u_{\varepsilon}^{-}=0$ for $0<\lambda<\lambda_{0}$. Hence $u_{\varepsilon} \geq 0$ a.e. in $\mathcal{O}$ for $\lambda \in\left(0, \lambda_{0}\right)$. By (2.16) this extend to all $\lambda>0$.

If we multiply the equation $(2.15)$ by $\beta\left(u_{\varepsilon}\right)$ and integrate on $\mathcal{O}$ we get via Green's formula that

$$
\begin{gathered}
\left(u_{\varepsilon}, \beta\left(u_{\varepsilon}\right)\right)_{2}+\lambda\left|\nabla \beta\left(u_{\varepsilon}\right)\right|_{2}^{2}+\lambda \varepsilon\left(\nabla u_{\varepsilon}, \nabla \beta\left(u_{\varepsilon}\right)\right)_{2} \\
+\lambda\left(\operatorname{div}\left(Y \alpha\left(u_{\varepsilon}\right)\right), \beta\left(u_{\varepsilon}\right)\right)_{2}=\left(f, \beta\left(u_{\varepsilon}\right)\right),
\end{gathered}
$$

and by (1.9) this yields

$$
C_{4}\left|u_{\varepsilon}\right|_{2}^{2}+\lambda\left|\nabla \beta\left(u_{\varepsilon}\right)\right|_{2}^{2} \leq \frac{C}{\lambda}|f|_{2}^{2}+C \lambda\left|\beta\left(u_{\varepsilon}\right)\right|_{2}^{2}+\frac{\lambda}{4}\left|\nabla \beta\left(u_{\varepsilon}\right)\right|_{2}^{2}
$$

where $C$ is independent of $\lambda$. 
We obtain that

$$
\left|u_{\varepsilon}\right|_{2}^{2}+\frac{\lambda}{2}\left|\nabla \beta\left(u_{\varepsilon}\right)\right|_{2}^{2} \leq \frac{C}{\lambda}|f|_{2}^{2}, \quad \forall \varepsilon>0,
$$

for $\lambda \in\left(0, \lambda_{0}\right)$, with $\lambda_{0}$ independent of $\varepsilon$.

Now if we multiply the equation by $u_{\varepsilon}$ and integrate on $\mathcal{O}$ we get by $(2.18)$ that $\left\{\varepsilon\left|\nabla u_{\varepsilon}\right|_{2}^{2}\right\}$ is bounded in $L^{2}(\mathcal{O})$.

By the compactness of $H_{0}^{1}(\mathcal{O})$ in $L^{2}(\mathcal{O})$ for $\varepsilon \rightarrow 0$, we have therefore on a subsequence again denoted $\left\{u_{\varepsilon}\right\}$ that

$$
\beta\left(u_{\varepsilon}\right) \rightarrow \zeta \text { strongly in } L^{2}(\mathcal{O}) \text { and weakly in } H_{0}^{1}(\mathcal{O}) .
$$

Combined with the fact that

$$
u_{\varepsilon} \rightarrow u \text { weakly in } L^{2}(\mathcal{O})
$$

and keeping in mind that the operator $u \rightarrow \beta(u)$ is $m$-accretive in $L^{2}(\mathcal{O})$, we have that $\beta(u)=\zeta$ a.e. on $\mathcal{O}$.

We have also that

$$
\alpha\left(u_{\varepsilon}\right) \rightarrow \alpha(u) \text { strongly in } L^{2}(\mathcal{O}) .
$$

Indeed, by (1.9), it follows that

$$
C_{6} \int_{\mathcal{O}}\left|\beta\left(u_{\varepsilon}\right)-\beta\left(u_{\lambda}\right)\right|^{2} d \xi \geq \int_{\mathcal{O}}\left|\alpha\left(u_{\varepsilon}\right)-\alpha\left(u_{\lambda}\right)\right|^{2} d \xi, \quad \forall \varepsilon, \lambda>0 .
$$

and since $\left\{\beta\left(u_{\varepsilon}\right)\right\}$ is strongly convergent in $L^{2}(\mathcal{O})$ we have that

$$
\left\{\alpha\left(u_{\varepsilon}\right)\right\} \text { is strongly convergent in } L^{2}(\mathcal{O}) .
$$

Taking into account that the operator $u \rightarrow \alpha(u)$ is maximal monotone in $L^{2}(\mathcal{O}) \times L^{2}(\mathcal{O})$, by $(2.20)$ and $(2.22)$ it follows $(2.21)$.

Using the above estimates, we can pass to the limit in

$$
u_{\varepsilon}-\lambda \Delta\left(\beta\left(u_{\varepsilon}\right)+\varepsilon u_{\varepsilon}\right)+\lambda \operatorname{div}\left(Y(\xi) \alpha\left(u_{\varepsilon}\right)\right)=f, \text { in } \mathcal{D}^{\prime}(\mathcal{O})
$$

and get therefore

$$
u-\lambda \Delta \beta(u)+\lambda \operatorname{div}(Y(\xi) \alpha(u))=f, \text { in } \mathcal{D}^{\prime}(\mathcal{O})
$$

for $f \in L^{2}(\mathcal{O})$ with $u \in L^{2}(\mathcal{O})$ and $\beta(u) \in H_{0}^{1}(\mathcal{O})$. Hence $u=u(\lambda, f)$.

Now letting $\varepsilon \rightarrow 0$ in (2.13) we get by (2.20) that

$$
\left|u^{1}-u^{2}\right|_{1} \leq\left|f_{1}-f_{2}\right|_{1}, \quad \forall f_{1}, f_{2} \in L^{2}(\mathcal{O}),
$$

because the functional $v \rightarrow \int_{\mathcal{O}}|v(\xi)| d \xi$ is convex and lower semicontinuous in $L^{2}(\mathcal{O})$. 
Finally, by approximating $f \in L^{2}(\mathcal{O})$ by a sequence $\left\{f_{n}\right\} \subset L^{1}(\mathcal{O})$ we get (2.24) for all $f_{1}, f_{2} \in L^{1}(\mathcal{O})$.

Taking into account that by $(2.24)$ we have

$$
\left|u_{n}-u_{m}\right|_{1} \leq\left|f_{n}-f_{m}\right|_{1} .
$$

Hence $u_{n} \rightarrow u$ in $L^{1}(\mathcal{O})$. Then by letting $n \rightarrow \infty$ in equation

$$
u_{n}-\lambda \Delta \beta\left(u_{n}\right)+\lambda \operatorname{div}\left(Y \alpha\left(u_{n}\right)\right)=f_{n}
$$

it follows by (2.19) and (2.21) that $u=u(\lambda, f)$, that is,

$$
u+\lambda A_{0} u=f
$$

and (2.24) extends to all $f \in L^{1}(\mathcal{O})$. Moreover letting $\varepsilon \rightarrow 0$ in (2.16) we get (2.5).

Assume now that $\alpha \in L^{\infty}(\mathbb{R})$ and prove that (2.8) and (2.9) holds. Here is the argument.

According to a classical result due to Stampacchia, for each $g=\left\{g_{i}\right\}_{i=0}^{d} \in$ $\left(L^{p}(\mathcal{O})\right)^{d+1}, p>d$, the boundary valued problem

$$
\begin{cases}-\Delta v=g_{0}+\sum_{i=1}^{d} \frac{\partial g_{i}}{\partial \xi_{i}}, & \text { in } \mathcal{O}, \\ v=0, & \text { on } \partial \mathcal{O},\end{cases}
$$

has a unique solution $v \in L^{\infty}(\mathcal{O}) \cap H_{0}^{1}(\mathcal{O})$ which satisfies

$$
|v|_{H_{0}^{1}(\mathcal{O})}+|v|_{\infty} \leq C \sum_{i=0}^{d}\left|g_{i}\right|_{p}
$$

By (2.25) we have via Green's formula that

$$
\lambda \int_{\mathcal{O}}\left(\nabla \beta\left(u_{n}\right)-Y \alpha\left(u_{n}\right)\right) \cdot \nabla v d \xi=\int_{\mathcal{O}}\left(f_{n}-u_{n}\right) v d \xi
$$

and this yields

$$
\begin{aligned}
& \lambda\left|\int_{\mathcal{O}} \nabla \beta\left(u_{n}\right) \cdot \nabla v d \xi\right| \\
\leq & \left(\left|f_{n}\right|_{1}+\left|u_{n}\right|_{1}\right)|v|_{\infty}+C \lambda|\nabla v|_{2} \\
\leq & 2\left|f_{n}\right|_{1}|v|_{\infty}+C \lambda|v|_{H_{0}^{1}(\mathcal{O})} \\
\leq & C\left|f_{n}\right|_{1} \sum_{i=0}^{d}\left|g_{i}\right|_{p}, \quad \forall g_{i} \in L^{p}(\mathcal{O}), i=0,1, \ldots, d,
\end{aligned}
$$

and by (2.26) and (2.28) we get

$$
\begin{aligned}
\lambda\left|\int_{\mathcal{O}}\left(g_{0} \beta\left(u_{n}\right)-g \cdot \nabla \beta\left(u_{n}\right)\right) d \xi\right| & \leq C\left|f_{n}\right|_{1} \sum_{i=0}^{d}\left|g_{i}\right|_{p}, \\
\forall g & =\left\{g_{i}\right\}_{i=0}^{d} \in\left(L^{p}(\mathcal{O})\right)^{d+1},
\end{aligned}
$$


and this implies that

$$
\left|\left\{\beta\left(u_{n}\right), \nabla \beta\left(u_{n}\right)\right\}\right|_{\left(L^{q}(\mathcal{O})\right)^{d+1}} \leq \frac{C}{\lambda}\left|f_{n}\right|_{1}, \quad \forall n, \lambda>0 .
$$

Hence

$$
\left|\beta\left(u_{n}\right)\right|_{W_{0}^{1, q}(\mathcal{O})} \leq \frac{C}{\lambda}\left|f_{n}\right|_{1}, \forall n, \lambda>0,
$$

where $\frac{1}{q}=1-\frac{1}{p}$ and $p>d$, that is $1<q<\frac{d}{d-1}$. Since $\beta\left(u_{n}\right) \rightarrow \beta(u)$ in $L^{1}(\mathcal{O})$ and $f_{n} \rightarrow f$ in $L^{1}(\mathcal{O})$ we infer that $\beta(u) \in W_{0}^{1, q}(\mathcal{O})$ and

$$
|\beta(u)|_{W_{0}^{1, q}(\mathcal{O})} \leq \frac{C}{\lambda}|f|_{1}, \forall \lambda>0 .
$$

Of course we have also $\alpha(u) \in W_{0}^{1,1}(\mathcal{O})$. This completes the proof of Theorem 1 .

Define the operator $A: D(A) \subset L^{1}(\mathcal{O}) \rightarrow L^{1}(\mathcal{O})$ by

$$
A u=A_{0} u, \text { for } \forall u \in D(A),
$$

where $D(A)=\left\{u=u(\lambda, v), v \in L^{1}(\mathcal{O})\right\}$. By $(2.5)$ we see that $D(A)$ is independent of $\lambda$.

As a consequence of Theorem 1, we obtain:

Theorem 2 Under hypotheses $i)$ the operator $A$ with the domain $D(A)$ is $m$-accretive in $L^{1}(\mathcal{O})$. Moreover,

$$
(I+\lambda A)^{-1} f=u(\lambda, f) \in\left(I+\lambda A_{0}\right)^{-1} f, \quad \forall \lambda>0,
$$

and $\overline{D(A)}=L^{1}(\mathcal{O})$. Furthermore, if $\alpha \in L^{\infty}(\mathbb{R})$ then

$$
\begin{aligned}
D(A)= & \left\{u \in L^{1}(\mathcal{O}) ; \beta(u) \in W_{0}^{1, q}(\mathcal{O}), 1 \leq q<\frac{d}{d-1},\right. \\
& \left.-\Delta \beta(u)+\operatorname{div}(Y \alpha(u)) \in L^{1}(\mathcal{O})\right\}
\end{aligned}
$$

Proof. By (2.31) it follows (2.32) while by (2.4) and (2.6) it is easily seen that $R(I+\lambda A)=L^{1}(\mathcal{O}), \lambda>0$ and that (1.12) holds, that is $A$ is $m$-accretive. To prove that $D(A)$ is dense in $L^{1}(\mathcal{O})$ we proceed as follows.

Let $f \in C_{0}^{\infty}(\mathcal{O})$ be arbitrary but fixed and let $f_{\varepsilon}$ be the solution to equation

$$
f_{\varepsilon}-\varepsilon \Delta \beta\left(f_{\varepsilon}\right)=f \text { in } \mathcal{O} ; \beta\left(f_{\varepsilon}\right) \in H_{0}^{1}(\mathcal{O}) .
$$

By elliptic regularity we have also $\beta\left(f_{\varepsilon}\right) \in H^{2}(\mathcal{O})$ and so multiplying $(2.33)$ by $\beta\left(f_{\varepsilon}\right)$ and $\Delta \beta\left(f_{\varepsilon}\right)$ respectively, and integrating on $\mathcal{O}$ we get

$$
\left|\nabla \beta\left(f_{\varepsilon}\right)\right|_{2}^{2}+\left|f_{\varepsilon}\right|_{2}^{2}+\varepsilon\left|\nabla \beta\left(f_{\varepsilon}\right)\right|_{2}^{2}+\varepsilon\left|\Delta \beta\left(f_{\varepsilon}\right)\right|_{2}^{2} \leq C|f|_{2}^{2} .
$$


Hence, for $\varepsilon \rightarrow 0$ we have

$$
\begin{aligned}
f_{\varepsilon} & \rightarrow f \text { strongly in } H^{-1}(\mathcal{O}) \\
& \text { weakly in } L^{2}(\mathcal{O}) \\
\beta\left(f_{\varepsilon}\right) & \rightarrow \beta(f) \text { weakly in } H_{0}^{1}(\mathcal{O}) \\
\varepsilon \Delta \beta\left(f_{\varepsilon}\right) & \rightarrow 0 \text { strongly in } H^{-1}(\mathcal{O}) .
\end{aligned}
$$

On the other hand, we have by $(2.33)$

$$
\int_{\mathcal{O}}\left|f_{\varepsilon}(x+h)-f_{\varepsilon}(x)\right| d x \leq \int_{\mathcal{O}}|f(x+h)-f(x)| d x, \forall h .
$$

Then, by Kolmogorov's compactness theorem it follows that $\left\{f_{\varepsilon}\right\}_{\varepsilon>0}$ is compact in $L^{1}(\mathcal{O})$ and therefore by $(2.35)$ we have

$$
f_{\varepsilon} \rightarrow f \text { strongly in } L^{1}(\mathcal{O}) \text { as } \varepsilon \rightarrow 0 .
$$

Now we rewrite (2.33) as

$$
f_{\varepsilon}+\varepsilon A_{0} f_{\varepsilon}=f-\varepsilon \operatorname{div}\left(Y \alpha\left(f_{\varepsilon}\right)\right)=g_{\varepsilon} .
$$

By (2.35) it follows that

$$
\begin{aligned}
& \left|\operatorname{div}\left(Y \alpha\left(f_{\varepsilon}\right)\right)\right|_{2} \\
\leq & |\operatorname{div}(Y)|_{\infty}\left|\alpha\left(f_{\varepsilon}\right)\right|_{2}+|Y|_{\infty}\left|\nabla \alpha\left(f_{\varepsilon}\right)\right|_{2} \leq C,
\end{aligned}
$$

because by hypotheses $i),\|\alpha\|_{L i p} \leq C\|\beta\|_{L i p}$ and so $\left\{\nabla \alpha\left(f_{\varepsilon}\right)\right\}$ is bounded in $L^{2}(\mathcal{O})$. Hence $g_{\varepsilon} \in \underline{L^{1}(\mathcal{O})}$ and therefore by $(2.37) f_{\varepsilon} \in D(\underline{A) \text {, while by }(2.36)}$ it follows that $f \in \overline{D(A)}$. Hence $C_{0}^{\infty}(\mathcal{O}) \subset \overline{D(A)}$ and so $\overline{D(A)}=L^{1}(\mathcal{O})$ as claimed.

Definition 3 The function $X:[0, T] \times \mathcal{O} \rightarrow \mathbb{R}$ is said to be a weak solution to equation (2.1) if $X \in C\left([0, T] ; L^{1}(\mathcal{O})\right)$ and $X(t)=\lim _{h \rightarrow 0} X_{h}(t)$ in $L^{1}(\mathcal{O})$, uniformly in $t \in[0, T]$, where

$$
\begin{gathered}
X_{h}(t)=X_{h}^{i}, t \in[i h,(i+1) h), i=0,1, \ldots, N-1, N h=T \\
\left\{\begin{array}{l}
X_{h}^{i+1}-h \Delta \beta\left(X_{h}^{i+1}\right)+h \operatorname{div}\left(Y \alpha\left(X_{h}^{i+1}\right)\right)=X_{h}^{i}+F_{h}^{i}, \quad \text { in } \mathcal{O} \\
X_{h}^{0}=X_{0},
\end{array}\right. \\
\text { and } X_{h}^{i} \in \widehat{D} \text { for } i=1,2, \ldots, N \text {. Here } F_{h}^{i}(\xi)=\int_{i h}^{(i+1) h} F(t, \xi) d t, \xi \in \mathcal{O} .
\end{gathered}
$$

We give now the main result of this section. 
Theorem 4 Assume that hypotheses $i)$ holds. Then for any $X_{0} \in L^{1}(\mathcal{O})$, $T>0$ and $F \in L^{1}((0, T) \times \mathcal{O})$ there is a unique weak solution $X=X\left(t, X_{0}\right) \in$ $C\left([0, T] ; L^{1}(\mathcal{O})\right)$ to equation (2.1).

Moreover, we have

$$
\left|X\left(t, X_{0}\right)-X\left(t, \overline{X_{0}}\right)\right|_{1} \leq\left|X_{0}-\overline{X_{0}}\right|_{1}, \forall t \in[0, T), \forall X_{0}, \overline{X_{0}} \in L^{1}(\mathcal{O}) .(2.40)
$$

Furtheron, if $X_{0} \geq 0$ a.e. in $\mathcal{O}$ and $F \geq 0$ a.e. on $(0, T) \times \mathcal{O}$ then $X \geq 0$ a.e. on $(0, T) \times \mathcal{O}$.

Furthermore for $F \equiv 0, t \longrightarrow X\left(t, X_{0}\right)$ is a $C_{0}$ semigroup of contractions in $L^{1}(\mathcal{O})$.

Proof. As mentioned earlier, we rewrite the equation as (2.3) where $A$ is defined by (2.31). Since $A$ is $m$-accretive in $L^{1}(\mathcal{O})$, we have by the Crandall and Liggett theorem (see e.g. [2], [10]) for each $X_{0} \in \overline{D(A)}=L^{1}(\mathcal{O})$ a unique mild solution $X \in C\left([0, T] ; L^{1}(\mathcal{O})\right)$.

This means that $X(t)=\lim _{h \rightarrow 0} X_{h}(t)$ in $L^{1}(\mathcal{O})$ where

$$
X_{h}(t)=Z_{h}^{i}, t \in[i h,(i+1) h), i=0,1, \ldots, N-1
$$

and

$$
Z_{h}^{i+1}+h A Z_{h}^{i+1}=Z_{h}^{i}+F_{h}^{i}, i=0,1, \ldots, N-1 .
$$

Taking into account (2.31) and the definition of $A_{0}$ we see that $Z_{h}^{i}=X_{h}^{i}$ in (2.38).

If $X_{0} \geq 0$ a.e. in $\mathcal{O}$ and $F \geq 0$ a.e. on $(0, T) \times \mathcal{O}$ then $F_{h}^{i} \geq 0 \forall i$ and so by (2.39) and (2.7) it follows that $X_{h}^{i+1} \geq 0, \forall i$ and so $X \geq 0$ as claimed.

We have also.

Theorem 5 If $X_{0} \in L^{2}(\mathcal{O})$ and $F \in L^{2}((0, T) \times \mathcal{O})$ then $X$ is a strong solution to (2.1) in the space $H^{-1}(\mathcal{O})$. More precisely, we have

$$
X \in L^{\infty}\left(0, T ; L^{2}(\mathcal{O})\right) \cap W^{1,2}\left([0, T] ; H^{-1}(\mathcal{O})\right), \beta(X) \in L^{2}\left(0, T ; H_{0}^{1}(\mathcal{O})\right)
$$

and $X:(0, T) \rightarrow L^{2}(\mathcal{O})$ is weakly continuous.

Proof. Formally, if we multiply $(2.1)$ by $\beta(X)$ and integrate on $(0, t) \times \mathcal{O}$ we get

$$
|X(t)|_{2}^{2}+\int_{0}^{t}|\nabla \beta(X(s))|_{2}^{2} d s \leq C\left(\left|X_{0}\right|_{2}^{2}+\int_{0}^{t}|F(s)|_{2}^{2} d s\right), \quad \forall t \in[0, T) .
$$

This formal calculation can be made rigorous by using the finite difference schema (2.38)-(2.39). Indeed if we multiply $(2.39)$ by $\beta\left(X_{h}^{i+1}\right)$ and integrate on $\mathcal{O}$ we get

$$
\begin{aligned}
& \int_{\mathcal{O}} g\left(X_{h}^{i+1}\right) d \xi+h \sum_{j=0}^{i+1}\left|\nabla \beta\left(X_{h}^{j}\right)\right|_{2}^{2} \\
\leq & C h \sum_{j=0}^{i+1}\left|X_{h}^{i+1}\right|_{2}^{2}+C \sum_{j=0}^{i+1}\left|F_{h}^{i}\right|_{2}^{2}
\end{aligned}
$$


where $g(r)=\int_{0}^{r} \beta(s) d s$. Then letting $h \rightarrow 0$ we get (2.41) as claimed.

Theorem 5 suggests an alternative approach to existence theory for equation (2.1) in the Sobolev space $H^{-1}(\mathcal{O})$ which as well known is another convenient space for nonlinear parabolic equations of porous media type (see e.g. [2], [3]). It should be said however that $L^{1}(\mathcal{O})$ is the natural space for this equation, not only for its physical significance for the problem, but mainly because only in this space the semigroup $S(t) X_{0}=X\left(t, X_{0}\right)$ is a semigroup of contractions and so the dynamics of the flow is dissipative on $(0, \infty)$. This fact is illustrated below.

\section{Long time behaviour of semigroup $S(t)$ that is:}

For each $X_{0} \in L^{1}(\mathcal{O})$ denote by $\Gamma\left(X_{0}\right)=\left\{S(t) X_{0} ; t \geq 0\right\}$ the orbit through $X_{0}$ of $S(t)$ and by $\omega\left(X_{0}\right)$ the corresponding $\omega$-limit set,

$$
\omega\left(X_{0}\right)=\left\{Z \in L^{1}(\mathcal{O}) ; Z=\lim _{t_{n} \rightarrow \infty} S\left(t_{n}\right) X_{0} \text { in } L^{1}(\mathcal{O})\right\} .
$$

We know by $(2.40)$ that $\Gamma\left(X_{0}\right)$ is bounded in $L^{1}(\mathcal{O})$.

Moreover, if $\alpha \in L^{\infty}(\mathcal{O})$, then for each $\lambda>0$ the operator $(I+\lambda A)^{-1}$ : $L^{1}(\mathcal{O}) \rightarrow L^{1}(\mathcal{O})$ is compact. Indeed, if $u=(I+\lambda A)^{-1} f$, where $f \in L^{1}(\mathcal{O})$ we have

$$
u-\lambda \Delta \beta(u)+\operatorname{div}(Y \alpha(u))=f, \text { in } \mathcal{D}^{\prime}(\mathcal{O}) .
$$

If $\alpha \in L^{\infty}(\mathcal{O})$, then by estimate (2.30) we know that

$$
|u|_{W_{0}^{1, q}(\mathcal{O})} \leq C|f|_{1}, \quad \forall f \in L^{1}(\mathcal{O}), 1<q<\frac{d}{d-1},
$$

and since $W_{0}^{1, q}(\mathcal{O})$ is compact in $L^{1}(\mathcal{O})$ we infer that $(I+\lambda A)^{-1}$ maps bounded subsets of $L^{1}(\mathcal{O})$ in precompact sets, as claimed. It is also clear that $0=A(0)$.

Then by Theorem 3 from [11] it follows that the orbit $\Gamma\left(X_{0}\right)$ of $S(t)$ is precompact in $L^{1}(\mathcal{O})$ and so $\omega\left(X_{0}\right) \neq \emptyset, \forall X_{0} \in L^{1}(\mathcal{O})$. It is also known that, $S(t)$ is an isometry on $\omega\left(X_{0}\right)$ and

$$
\omega\left(X_{0}\right) \subset\left\{y \in L^{1}(\mathcal{O}) ;\left|y-y_{0}\right|_{1}=r\right\},
$$

where $S(t) y_{0}=y_{0}$.

Theorem 6 , which follows, makes precise the structure of $\omega\left(X_{0}\right)$. For simplicity we assume that $X_{0} \geq 0$, which implies that $X\left(t, X_{0}\right) \geq 0, \forall t \geq 0, \xi \in \mathcal{O}$.

Theorem 6 Assume that hypotheses i) hold and

$$
\begin{gathered}
Y=-\nabla g, g \in W^{1, \infty}(\mathcal{O}), g \geq 0 \text { a.e. in } \mathcal{O}, \alpha \in L^{\infty}(\mathbb{R}) \\
\liminf _{r \rightarrow 0^{+}} \frac{\alpha(r)}{r}=r_{0}>0 \\
\beta \in \operatorname{Lip}(\mathbb{R}) .
\end{gathered}
$$


Let $X_{0} \in L^{2}(\mathcal{O})$ be such that $X_{0} \geq 0$ a.e. in $\mathcal{O}$. Then

$$
\omega\left(X_{0}\right) \subset\left\{Z \in L^{1}(\mathcal{O}) ; \nabla \beta(Z)-\alpha(Z) Y=0 \text {, a.e. in } \mathcal{O}\right\} \text {. }
$$

In particular, it follows by (2.45) that if the equation $\nabla \beta(Z)-\alpha(Z) Y=$ 0 , a.e. in $\mathcal{O}$ has a unique solution then there is $\lim _{t \rightarrow \infty} S(t) X_{0}=X_{\infty}$ in $L^{1}(\mathcal{O})$.

Proof. We shall use an argument from [6]. We consider the function $V$ : $\left.\left.L^{1}(\mathcal{O}) \rightarrow \overline{\mathbb{R}}=\right]-\infty,+\infty\right]$ and $G: L^{1}(\mathcal{O}) \rightarrow \mathbb{R}$ defined by

$$
\begin{gathered}
V(u)=\int_{\mathcal{O}}(\zeta(u(\xi))+g(\xi) u(\xi)) d \xi \\
G(u)=\int_{\mathcal{O}}\left|\frac{\nabla \beta(u(\xi))}{\sqrt{\alpha(u(\xi))}}+\sqrt{\alpha(u(\xi))} \nabla g(\xi)\right|^{2} d \xi
\end{gathered}
$$

where

$$
\zeta(r)=-\int_{0}^{r} d s \int_{s}^{1} \frac{\beta^{\prime}(s)}{\alpha(s)} d s, \forall r \in \mathbb{R} .
$$

We note that by $(2.44), \beta^{\prime} \in L_{l o c}^{1}(\mathbb{R})$.

It is easily seen that $V$ with the domain $D(V)=\left\{u \in L^{1}(\mathcal{O}) ; V(u)<\infty\right\}$ is lower-semicontinuous. As regards $G$ we can rewrite it as

$$
\begin{aligned}
G(u) & =\int_{\mathcal{O}}\left|\frac{\beta^{\prime}(u(\xi)) \nabla(u(\xi))}{\sqrt{\alpha(u(\xi))}}+\sqrt{\alpha(u(\xi))} \nabla g(\xi)\right|^{2} d \xi \\
& =\int_{\mathcal{O}}|\nabla j(u(\xi))+\sqrt{\alpha(u(\xi))} \nabla g(\xi)|^{2} d \xi
\end{aligned}
$$

where $j(r)=\int_{0}^{r} \frac{\beta^{\prime}(s)}{\sqrt{\alpha(s)}} d s \leq C \sqrt{r}, \forall r \geq 0$.

This implies that $G$ is lower-semicontinuous too on $L^{1}(\mathcal{O})$. Moreover, we note that $V$ is convex in $L^{2}(\mathcal{O})$ and its differential $\nabla V$ is given by

$$
\nabla V(u)=-\int_{u}^{1} \frac{\beta^{\prime}(s)}{\alpha(s)} d s+g, \forall u \in L^{2}(\mathcal{O}) .
$$

We note also that

$$
(A u, \nabla V(u))_{2} \geq G(u), \forall u \in D(A) \cap L^{2}(\mathcal{O}),
$$

which means that $V$ is a Lyapunov function for semigroup $S(t)$.

The latter follows first via equation (1.10) for $A_{\varepsilon}$ and let after $\varepsilon$ go to zero. If we multiply equation

$$
u+\lambda A u=f
$$

(equivalently $u+\lambda A_{0} u=f$ ) by $\nabla V(u)$ scalarly in $L^{2}(\mathcal{O})$, it follows by $(2.46)$ that

$$
V\left((I+\lambda A)^{-1} f\right)+\lambda G\left((I+\lambda A)^{-1} f\right) \leq V(f), \quad \forall \lambda>0, f \in L^{2}(\mathcal{O}) .
$$

This implies in particular that $V$ is a Lyapunov function for the semigroup $S(t)$ and by Theorem 4.1 in [17] we it follows that $\omega\left(X_{0}\right)=\left\{Z \in L^{1}(\mathcal{O}) ; G(Z)=0\right\}$ and so (2.45) follows. 


\section{Phase transition with non-degenerated enthalpy and convection from the Navier-Stokes equa- tion}

In this section, we study the same physical problem by using a different mathematical model. More precisely, we consider a phase transition problem, in a bounded domain $\mathcal{O} \in \mathbb{R}^{3}$ where the convection in the liquid phase is modelled by the Navier-Stokes equation. In this context, we need, for technical reasons, to take a regularized form of the enthalpy function and therefore the result from this section is not a generalization of the previous case.

In the construction of the model, we replace the Heaviside function by a $C^{1}$ monotone function like, for example

$$
\widetilde{H}(r)= \begin{cases}0, & r<0, \\ \varphi(r), & r \in[0, \mu], \\ 1, & r>\mu,\end{cases}
$$

where $\varphi \in C^{2}[0, \mu], \varphi^{\prime}>0$ on $(0, \mu), \varphi(0)=0, \varphi(\mu)=1$ and $\varphi^{\prime}(0)=0$ and $\varphi^{\prime}(\mu)=0$.

Consequently, the enthalpy function (1.3) becomes in this case

$$
\gamma(r)=C(r)+l \widetilde{H}(r) .
$$

One can easily see that $\gamma$ is Lipschitz and therefore $\gamma^{-1}$ is strongly monotone (i.e. it's derivative is bounded from below by a positive constant).

By arguing as in the introduction we obtain the equation

$$
\begin{cases}\frac{\partial X}{\partial t}-\Delta \Psi(X)+\operatorname{div}\left(Y \eta\left(\gamma^{-1}(X)\right)\right)=F, & \text { in }(0, T) \times \mathcal{O} \\ X(0, \xi)=X_{0}(\xi), & \text { in } \mathcal{O}, \\ X(t, \xi)=0, & \text { on } \partial \mathcal{O},\end{cases}
$$

with smooth enthalpy. Here $Y=Y(t, \xi)$ on $(0, T) \times \mathcal{O}$.

Keeping in mind the form of $\Psi$ i.e.,

$$
\Psi(r)=K\left(\gamma^{-1}(r)\right)
$$

where

$$
K(r)= \begin{cases}k_{1} r, & r \leq 0 \\ k_{2} r, & r>0,\end{cases}
$$

and the fact that $k_{1}$ and $k_{2}$ are positive constants, we get by elementary calculus that $\Psi$ is also strongly increasing, which means that there exists a positive constant $\psi_{0}$ such that

$$
(\Psi(x)-\Psi(y))(x-y) \geq \psi_{0}|x-y|^{2}, \quad \forall x, y \in \mathbb{R} .
$$


We have also that $\Psi$ is Lipschitz, $\Psi(0)=0$ and in particular $\Psi(x) x \geq$ $\psi_{0}|x|^{2}, \forall x \in \mathbb{R}$.

As in the first part of the paper, $\eta$ is assumed to be a Lipschitz monotonically non decreasing function such that $\eta(r)=0, \forall r \leq 0$. The heating source $F$ is now assumed to be $L^{2}\left(\left(0, T^{*}\right) \times \mathcal{O}\right)$.

The main difference with respect to the previous case comes from the fact that the velocity $Y$ from the convection term is the solution of a Navier-Stokes equation.

More precisely we are interested in the following system

$$
\begin{cases}\frac{\partial X}{\partial t}-\Delta \Psi(X)+\operatorname{div}\left(Y \eta\left(\gamma^{-1}(X)\right)\right)=F, & \text { in }(0, T) \times \mathcal{O}, \\ X(0, \xi)=X_{0}(\xi), & \text { in } \mathcal{O}, \\ X(t, \xi)=0, & \text { on }(0, T) \times \partial \mathcal{O}, \\ \frac{\partial Y}{\partial t}-\nu \Delta Y+(Y \cdot \nabla) Y-f_{B}\left(\gamma^{-1}(X)\right)=-\nabla p & \\ +g\left(\gamma^{-1}(X)\right) Y, & \text { in }(0, T) \times \mathcal{O}, \\ \nabla \cdot Y=0, & \text { in }(0, T) \times \mathcal{O} \\ Y(0)=Y_{0}, & \text { in } \mathcal{O}, \\ Y=0, & \text { on }(0, T) \times \partial \mathcal{O} .\end{cases}
$$

The vectorial function $Y=\left(Y_{1}, Y_{2}, Y_{3}\right)$ which now is time dependent and solves the second equation of the system is interpreted as the velocity field of a viscous, incompressible Newtonian fluid. As usually, we denote by $p$ the pressure and by $\nu>0$ the viscosity of the fluid. The buoyancy force $f_{B}$ is a function of Boussinesq type which is assumed to be Lipschitz. The function $g$ is a penalty form of Carman-Kozeny type that is added to bring the velocity to zero in the solid phase:

$$
g(r)=-\frac{C_{C K}(1-\tau(r))^{2}}{(\tau(r))^{3}+C},
$$

where $\tau(r)=\left\{\begin{array}{ll}1, & r \geq 0, \\ 0, & r<0,\end{array}\right.$ is the liquid fraction, $C_{C K}$ the Carman-Kozeny constant (usually set to a large value). The constant $C$ is introduced to avoid divisions by zero. By an elementary calculus we get that

$$
g(r)=\left\{\begin{array}{cc}
0, & r \geq 0 \\
-\frac{C_{C K}}{C}, & r<0,
\end{array}\right.
$$

which means that the corresponding term disappears in the liquid phase and becomes a large constant compensating all the other terms in the solid phase.

This function, combined with $\eta$ from the previous equation, which disappears in the solid phase, ensures that the convection term appears only in the liquid phase. Note that $g$ is a Lipschitz, bounded function. 
In the second equation of the system, we have used the following standard notation

$$
(Y \cdot \nabla) Y=\sum_{i=1}^{3} Y_{i} D_{i} Y_{j}, \quad \forall j=\overline{1,3}
$$

We aim to prove existence and uniqueness of the solution for the previous system by using a fixed point approach.

To this purpose, we first fix $X \in L^{2}((0, T) \times \mathcal{O})$ and prove, by adapting a classical approach for Navier-Stokes equation, the well-posedness for the following problem

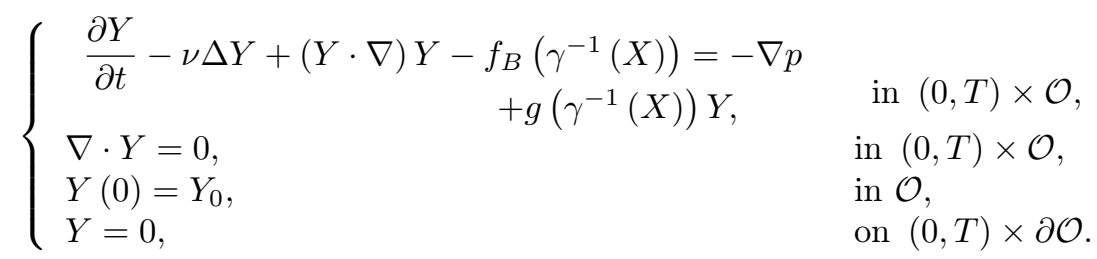

We start by recalling some notations.

Let

$$
H=\left\{Y \in\left(L^{2}(\mathcal{O})\right)^{3} ; \nabla \cdot Y=0, Y \cdot n=0 \text { on } \partial \mathcal{O}\right\},
$$

where $n$ is the outward normal to $\partial \mathcal{O}$ and

$$
V=\left\{Y \in\left(H_{0}^{1}(\mathcal{O})\right)^{3} ; \nabla \cdot Y=0\right\}=H \cap\left(H_{0}^{1}(\mathcal{O})\right)^{3} .
$$

Note that $H$ is a closed subspace of $\left(L^{2}(\mathcal{O})\right)^{3}$ and is a Hilbert space with the scalar product of $\left(L^{2}(\mathcal{O})\right)^{3}$.

We denote by

$$
P:\left(L^{2}(\mathcal{O})\right)^{3} \longrightarrow H
$$

the Leray projector that is the orthogonal projection of $\left(L^{2}(\mathcal{O})\right)^{3}$ onto $H$. We use it to construct the Stokes operator

$$
\widetilde{A}: V \longrightarrow V^{\prime} \text { defined by } \widetilde{A}(Y)=-P(\Delta Y) \text {. }
$$

The domain of $\widetilde{A}$ in $H$ is $D(\widetilde{A})=\left(H^{2}(\mathcal{O})\right)^{3} \cap V$. Define also the nonlinear operator

$$
B: V \rightarrow V^{\prime} \text { defined by } B(Y)=P((Y \cdot \nabla Y) Y),
$$

where $V^{\prime}$ is the dual of $V$.

By applying the Leray projector to (3.5) we get the $3 D$ Navier-Stokes equation operatorial form

$$
\left\{\begin{array}{l}
\frac{d Y}{d t}+\nu \widetilde{A}(Y)+B(Y)-P\left(f_{B}\left(\gamma^{-1}(X)\right)\right)=P\left(g\left(\gamma^{-1}(X)\right) Y\right), \quad(0, T), \\
Y(0)=Y_{0} .
\end{array}\right.
$$

We have the following existence result for the equation above. 
Theorem 7 For each $Y_{0} \in D(\widetilde{A})$ and $X \in L^{2}((0, T) \times \mathcal{O})$ there is a unique function $Y \in W^{1,2}\left(\left[0, T^{*}\right] ; H\right) \cap L^{2}\left(0, T^{*} ; D(\widetilde{A})\right) \cap C\left(\left[0, T^{*}\right] ; V\right)$ which satisfies (3.6) on $\left(0, T^{*}\right)$ for some $T^{*}=T^{*}\left(\left|Y_{0}\right|_{V}\right)<T$. In $2-D$ we have $T=T^{*}$. Moreover, the map $X \rightarrow Y$ is continuous from $L^{2}\left(\left(0, T^{*}\right) \times \mathcal{O}\right)$ to $L^{2}(0, T ; H)$.

Proof. We set

$$
G(X)=P\left(f_{B}\left(\gamma^{-1}(X)\right)\right)
$$

and define $P_{X(t)}:\left(L^{2}(\mathcal{O})\right)^{3} \rightarrow H$

$$
P_{X(t)}(Y)=P\left(g\left(\gamma^{-1}(X(t)) Y\right)\right) .
$$

One can easily see that for each $X \in L^{2}((0, T) \times \mathcal{O})$ fixed, by using the fact that $g$ is bounded, we have that the operator

$$
P_{X(t)}: L^{2}(0, T ; H) \rightarrow L^{2}(0, T ; H)
$$

is well defined and Lipschitz.

Since $f_{B} \circ \gamma^{-1}$ is Lipschitz, $G$ is also Lipschitz.

We consider now the equation

$$
\left\{\begin{array}{l}
\frac{d Y}{d t}+\nu \widetilde{A}(Y)+B(Y)-P_{X(t)}(Y)=G(X), \quad(0, T), \\
Y(0)=Y_{0}
\end{array}\right.
$$

and for the proof of existence, we approximate the operator $B$ which is continuous and locally Lipschitz by the following Lipschitz operator. For each $M>0$ we define

$$
B_{M}(Y)=\left\{\begin{array}{cc}
B(Y), & |Y|_{V} \leq M, \\
B\left(\frac{M Y}{\|Y\|_{V}}\right), & |Y|_{V}>M .
\end{array}\right.
$$

We define $\Gamma_{M}: D\left(\Gamma_{M}\right) \subset H \rightarrow H$ by

$$
\Gamma_{M}=\nu \widetilde{A}+B_{M}, \text { for } D\left(\Gamma_{M}\right)=D(\widetilde{A}) .
$$

By the classical properties of $\widetilde{A}$ and $B$, and considering that $\left|P_{X(t)}(Y)\right|_{H} \leq$ $C|Y|_{H}$ we see that $\Gamma_{M}$ is well defined.

We have also (see [2], page 254):

Lemma 8 The operator $\Gamma_{M}$ is quasi-m-accretive in $H \times H$.

We continue now the proof of the theorem.

Since by the previous lemma the operator $\Gamma_{M}$ generates a semi-group of quasi-contractions on $H$ and $P_{X(t)}$ is Lipschitz, follows that the equation

$$
\left\{\begin{array}{l}
\frac{d Y_{M}}{d t}+\nu \widetilde{A}\left(Y_{M}\right)+B_{M}\left(Y_{M}\right)-P_{X(t)}\left(Y_{M}\right)=G(X), \quad(0, T), \\
Y(0)=Y_{0}
\end{array}\right.
$$


has a unique solution

$$
Y_{M} \in C([0, T] ; V) \cap L^{2}(0, T ; D(\widetilde{A})) .
$$

To complete the proof, it is enough to show that for $M$ sufficiently large, the flow $Y_{M}$ is independent of $M$ on a certain interval [0,T $\left.\left(Y_{0}\right)\right]$.

First we multiply (3.7) by $Y_{M}$ and integrate on $(0, t)$. We have

$$
\begin{aligned}
& \left|Y_{M}\right|_{H}^{2}+\nu \int_{0}^{t}\left|Y_{M}\right|_{V}^{2} d s \\
\leq & C\left(\left|Y_{0}\right|_{H}^{2}+\frac{1}{2 \nu} \int_{0}^{t}|G(X)|_{H}^{2} d s\right)+\int_{0}^{t}\left\langle P_{X(t)}\left(Y_{M}\right), Y_{M}\right\rangle_{H} d s \\
\leq & C\left(\left|Y_{0}\right|_{H}^{2}+\frac{1}{2 \nu} \int_{0}^{t}|G(X)|_{H}^{2} d s\right)+C \int_{0}^{t}\left|Y_{M}\right|_{H}^{2} d s,
\end{aligned}
$$

and by Gronwall's lemma we obtain that

$$
\left|Y_{M}\right|_{H}^{2}+\nu \int_{0}^{t}\left|Y_{M}\right|_{V}^{2} d s \leq C\left(\left|Y_{0}\right|_{H}^{2}+\frac{1}{2 \nu} \int_{0}^{t}|G(X)|_{H}^{2} d s\right) .
$$

Next we multiply (3.7) by $\widetilde{A}\left(Y_{M}\right)$ and we get that

$$
\begin{aligned}
& \frac{1}{2} \frac{d}{d t}\left|Y_{M}\right|_{V}^{2}+\nu\left|\widetilde{A}\left(Y_{M}\right)\right|_{H}^{2} \\
\leq & \left|\left\langle P_{X(t)}\left(Y_{M}\right), \widetilde{A}\left(Y_{M}\right)\right\rangle_{H}\right|+\left|\left\langle B_{M}\left(Y_{M}\right), \widetilde{A}\left(Y_{M}\right)\right\rangle_{H}\right|+|G(X)|_{H}\left|\widetilde{A}\left(Y_{M}\right)\right|_{H} .
\end{aligned}
$$

This yields

$$
\begin{aligned}
& \left|Y_{M}\right|_{V}^{2}+\nu \int_{0}^{t}\left|\widetilde{A}\left(Y_{M}\right)\right|_{H}^{2} d s \\
\leq & \int_{0}^{t}\left|\left\langle P_{X(t)}\left(Y_{M}\right), \widetilde{A}\left(Y_{M}\right)\right\rangle_{H}\right| d s+\int_{0}^{t}\left|\left\langle B_{M}\left(Y_{M}\right), \widetilde{A}\left(Y_{M}\right)\right\rangle_{H}\right| d s \\
& +C\left(\left|Y_{0}\right|_{V}^{2}+\frac{1}{2 \nu} \int_{0}^{t}|G(X)|_{H}^{2} d s\right) \\
\leq & \int_{0}^{t}\left(\frac{1}{\nu}\left|P_{X(t)}\left(Y_{M}\right)\right|_{H}^{2}+\frac{\nu}{4}\left|\widetilde{A}\left(Y_{M}\right)\right|_{H}^{2}\right) d s \\
& +\int_{0}^{t}\left(\frac{1}{\nu}\left|B_{M}\left(Y_{M}\right)\right|_{H}^{2}+\frac{\nu}{4}\left|\widetilde{A}\left(Y_{M}\right)\right|_{H}^{2}\right) d s \\
& +C\left(\left|Y_{0}\right|_{V}^{2}+\frac{1}{2 \nu} \int_{0}^{t}|G(X)|_{H}^{2} d s\right) .
\end{aligned}
$$


We obtain that

$$
\begin{aligned}
& \left|Y_{M}\right|_{V}^{2}+\frac{\nu}{2} \int_{0}^{t}\left|\widetilde{A}\left(Y_{M}\right)\right|_{H}^{2} d s \\
\leq & \int_{0}^{t} \frac{1}{\nu}\left|P_{X(t)}\left(Y_{M}\right)\right|_{H}^{2} d s+\int_{0}^{t} \frac{1}{\nu}\left|B_{M}\left(Y_{M}\right)\right|_{H}^{2} d s \\
& +C\left(\left|Y_{0}\right|_{V}^{2}+\frac{1}{2 \nu} \int_{0}^{t}|G(X)|_{H}^{2} d s\right) \\
\leq & C\left(\left|Y_{0}\right|_{V}^{2}+\frac{1}{2 \nu} \int_{0}^{t}|G(X)|_{H}^{2} d s+\frac{1}{\nu} \int_{0}^{t}\left|Y_{M}\right|_{H}^{2} d s+\int_{0}^{t}\left|Y_{M}\right|_{V}^{6} d s\right) .
\end{aligned}
$$

By using (3.8) we get

$$
\begin{aligned}
& \left|Y_{M}\right|_{V}^{2}+\frac{\nu}{2} \int_{0}^{t}\left|\widetilde{A}\left(Y_{M}\right)\right|_{H}^{2} d s \\
\leq & C\left(\left|Y_{0}\right|_{V}^{2}+\int_{0}^{t}|G(X)|_{H}^{2} d s+\int_{0}^{t}\left|Y_{M}\right|_{V}^{6} d s\right) .
\end{aligned}
$$

Momentarily dropping the term $\frac{\nu}{2} \int_{0}^{t}\left|\widetilde{A}\left(Y_{M}\right)\right|_{H}^{2} d s$ we have a differential inequality of the type

$$
\varphi^{\prime} \leq \frac{C}{\nu} \varphi^{3}, \quad \forall t \in(0, T), \varphi(0)=C\left(\left|Y_{0}\right|_{V}^{2}+\int_{0}^{T}|X(s)|_{2}^{2} d s\right)
$$

This yields

$$
\left|Y_{M}(t)\right|_{V}^{2} \leq \frac{\nu \varphi^{2}(0)}{\nu-2(C \varphi(0))^{2} t}, \forall t \in\left[0, \frac{\nu}{2(C \varphi(0))^{2}}\right) .
$$

Hence

$$
\left|Y_{M}(t)\right|_{V}^{2} \leq\left(\frac{\nu}{2 \delta C}\right)^{\frac{1}{2}}, \quad \forall t \in\left[0, T^{*}-\delta\right)
$$

where

$$
T^{*}=\frac{\nu}{2\left(C\left(\left|Y_{0}\right|_{V}^{2}+\int_{0}^{T}|X(s)|_{2}^{2} d s\right)\right)}, \forall \delta \in\left(0, T^{*}\right) .
$$

This leads to

$$
\begin{aligned}
& \left|Y_{M}\right|_{V}^{2}+\frac{\nu}{2} \int_{0}^{t}\left|\widetilde{A}\left(Y_{M}\right)\right|_{H}^{2} d s \\
\leq & C(\varepsilon)\left(\left|Y_{0}\right|_{V}^{2}+\int_{0}^{t}|G(X)|_{H}^{2} d s\right), \quad \forall t \in\left(0, T^{*}-\varepsilon\right), \forall \varepsilon>0 .
\end{aligned}
$$

Hence $B_{M} Y_{M}=B Y_{M}$ on $\left(0, T^{*}\right)$ and therefore $Y_{M}=Y$ and this completes the proof of the existence. In $2 D$ we have $T^{*}=T$. 
The uniqueness is direct from the argument used in [2] and the Lipschitz property of $P_{X(t)}$.

In particular, it follows that

$$
\left|Y_{M}(t)\right|_{V} \leq C\left(\left|Y_{0}\right|_{V}^{2}+\int_{0}^{T}|X(s)|_{2}^{2} d s\right)^{\frac{1}{2}}, \quad \forall t \in\left[0, T^{*}\right]
$$

for $T^{*} \leq \frac{C}{\left|Y_{0}\right|_{V}^{2}+\int_{0}^{T}|X(s)|_{2}^{2} d s}$ where $C$ is independent of $Y_{0}$ and $T$.

By the above estimates it follows also that if $X_{n} \rightarrow X$ in $L^{2}((0, T) \times \mathcal{O})$ then $Y_{n}=Y\left(X_{n}\right) \rightarrow Y(X)$ in $L^{2}(0, T ; H)$.

We shall continue now with the study of the first equation of the system. To this purpose we denote by $\widetilde{Y}=\widetilde{Y}_{X}(t)$ the solution of the second equation for the function $X \in L^{2}((0, T) \times \mathcal{O})$ fixed before, and we replace it in the first equation.

Note that, by the Sobolev embedding theorem (see e.g. Corollary 9.13 from [9]) we have that $W^{2,2}(\mathcal{O}) \subset L^{\infty}(\mathcal{O})$ for $\mathcal{O} \subset \mathbb{R}^{3}$. From the properties of the solution $\widetilde{Y}$ we have therefore that $\widetilde{Y} \in L^{2}\left(0, T^{*} ;\left(L^{\infty}(\mathcal{O})\right)^{3}\right)$.

Consider the space $\left(L^{2}(\mathcal{O})\right)^{*}=\mathcal{Z}$ which is the dual of the space $L^{2}(\mathcal{O}) \subset$ $H^{-1}(\mathcal{O})$ in the pairing $\langle., .\rangle_{-1}$ defined by $H^{-1}(\mathcal{O})$.

In other words, the spaces $L^{2}(\mathcal{O})$ and $\mathcal{Z}$ are in duality with pivot space $H^{-1}(\mathcal{O})$.

We have $\mathcal{Z}=\left\{z=-\Delta y ; y \in L^{2}(\mathcal{O})\right\}$ and $L^{2}(\mathcal{O}) \subset H^{-1}(\mathcal{O}) \subset \mathcal{Z}$ with continuous and dense embeddings where

$$
L^{2}(\mathcal{O})\langle u, z\rangle_{\mathcal{Z}}=\int_{\mathcal{O}}(-\Delta)^{-1} z u d \xi, \forall u \in L^{2}(\mathcal{O}), z \in \mathcal{Z} .
$$

Define the operator $A(t): L^{2}(\mathcal{O}) \rightarrow \mathcal{Z}, \forall t \in\left(0, T^{*}\right)$ by

$$
A(t) u=-\Delta \Psi(u)+\operatorname{div}\left(\widetilde{Y}(t) \eta\left(\gamma^{-1}(u)\right)\right), \quad \forall u \in L^{2}(\mathcal{O}) .
$$

We note that

$$
\begin{aligned}
& \mathcal{Z}(A(t)(u)-A(t)(v), u-v)_{L^{2}(\mathcal{O})} \\
= & (\Psi(u)-\Psi(v), u-v)_{2} \\
& +\left(\operatorname{div}\left(\tilde{Y}(t) \eta\left(\gamma^{-1}(u)\right)-\tilde{Y}(t) \eta\left(\gamma^{-1}(v)\right)\right), u-v\right)_{-1} \\
\geq & \psi_{0}|u-v|_{2}^{2}-\frac{\psi_{0}}{2}|u-v|_{2}^{2}-\frac{|\widetilde{Y}(t)|_{\infty} L}{2 \psi_{0}}|u-v|_{-1}^{2} \\
\geq & \frac{\psi_{0}}{2}|u-v|_{2}^{2}-\alpha(t)|u-v|_{-1}^{2},
\end{aligned}
$$

where $\alpha(t)=\frac{L}{2 \Psi_{0}}|\widetilde{Y}(t)|_{\infty}, t \in\left(0, T^{*}\right)$. 
By the previous computation we have also

$$
\mathcal{Z}(A(t)(u), u)_{L^{2}(\mathcal{O})} \geq \frac{\psi_{0}}{2}|u|_{2}^{2}-\alpha(t)|u|_{-1}^{2}, \quad \forall u \in L^{2}(\mathcal{O}), t \in\left[0, T^{*}\right] .
$$

Note also that

$$
|A(t)(u)|_{\mathcal{Z}}=\sup _{\varphi \in L^{2}(\mathcal{O}),|\varphi|_{2} \leq 1} \mathcal{Z}(A(t)(u), \varphi)_{L^{2}(\mathcal{O})} \leq C \alpha(t)|u|_{2}^{2},
$$

for $\forall u \in L^{2}(\mathcal{O}), t \in\left[0, T^{*}\right]$.

We note also that for each $t, A(t): L^{2}(\mathcal{O}) \rightarrow \mathcal{Z}$ is demicontinuous, i.e. strongly-weakly continuous.

Lemma 9 For each $X_{0} \in H^{-1}(\mathcal{O})$ and $F \in L^{2}\left(0, T^{*} ; L^{2}(\mathcal{O})\right)$ that is a unique solution

$$
X \in C\left(\left[0, T^{*}\right] ; H^{-1}(\mathcal{O})\right) \cap L^{2}\left(0, T^{*} ; L^{2}(\mathcal{O})\right) \cap W^{1,1}\left(\left[0, T^{*}\right] ; \mathcal{Z}\right)
$$

to the Cauchy problem

$$
\left\{\begin{array}{l}
\frac{d X}{d t}+A(t) X=F, \quad t \in\left(0, T^{*}\right), \\
X(0)=X_{0} .
\end{array}\right.
$$

Proof. We approximate (3.13) by

$$
\left\{\begin{array}{l}
\frac{d X_{\varepsilon}}{d t}+A_{\varepsilon}(t) X_{\varepsilon}=F, \quad t \in\left(0, T^{*}\right), \\
X_{\varepsilon}(0)=X_{0}
\end{array}\right.
$$

where

$$
A_{\varepsilon}(t) u=-\Delta \Psi(u)+\operatorname{div}\left(\widetilde{Y}_{\varepsilon}(t) \eta\left(\gamma^{-1}(u)\right)\right)
$$

and

$$
\widetilde{Y}_{\varepsilon}(t)=(\varepsilon \alpha(t)+1)^{-1} \tilde{Y}(t), \quad \varepsilon>0 .
$$

By (3.10)-(3.12), for each $\varepsilon>0$, the operator

$$
A_{\varepsilon}(t): L^{2}(\mathcal{O}) \rightarrow \mathcal{Z}
$$

is quasi-monotone, that is

$$
\mathcal{Z}\left(A_{\varepsilon}(t) u-A_{\varepsilon}(t) v, u-v\right)_{2} \geq \frac{\psi_{0}}{2}|u-v|_{2}^{2}-C_{\varepsilon}|u-v|_{-1}^{2}, \forall u, v \in L^{2}(\mathcal{O})
$$

and also coercive. Moreover, we have

$$
\left|A_{\varepsilon}(t) u\right|_{\mathcal{Z}} \leq C|u|_{L^{2}(\mathcal{O})}, \forall u \in L^{2}(\mathcal{O}), t \in\left[0, T^{*}\right) .
$$


Hence by Theorem 4.17 in [2], (3.14) has a unique solution

$$
X_{\varepsilon} \in C\left(\left[0, T^{*}\right] ; H^{-1}(\mathcal{O})\right) \cap L^{2}\left(0, T^{*} ; L^{2}(\mathcal{O})\right) \cap W^{1,2}\left(\left[0, T^{*}\right] ; \mathcal{Z}\right) .
$$

We have the estimate

$$
\begin{gathered}
\left|X_{\varepsilon}(t)\right|_{-1}^{2}+\int_{0}^{t}\left|X_{\varepsilon}(s)\right|_{2}^{2} d s \\
\leq\left|X_{0}\right|_{-1}^{2}+C \int_{0}^{t}\left|X_{\varepsilon}(s)\right|_{-1}^{2} \alpha(s) d s .
\end{gathered}
$$

Hence by Gronwall inequality we get

$$
\left|X_{\varepsilon}(t)\right|_{-1}^{2}+\int_{0}^{t}\left|X_{\varepsilon}(s)\right|_{2}^{2} d s \leq C_{1}\left|X_{0}\right|_{-1}^{2} \exp \left(\int_{0}^{t} \alpha(s) d s\right) .
$$

Similarly we get for $X_{\varepsilon}-X_{\lambda}, \forall \varepsilon, \lambda>0$, the estimate

$$
\begin{aligned}
& \left|X_{\varepsilon}(t)-X_{\lambda}(t)\right|_{-1}^{2}+\int_{0}^{t}\left|X_{\varepsilon}(t)-X_{\lambda}(t)\right|_{2}^{2} d s \\
\leq & C \int_{0}^{t}\left|\widetilde{Y}_{\varepsilon}(s)-\tilde{Y}_{\lambda}(s)\right|_{\infty}\left|X_{\varepsilon}(s)\right|_{-1}\left|X_{\varepsilon}(s)\right|_{2} \alpha(s) d s
\end{aligned}
$$

and this yields

$$
\left|X_{\varepsilon}(t)-X_{\lambda}(t)\right|_{-1}^{2}+\int_{0}^{t}\left|X_{\varepsilon}(t)-X_{\lambda}(t)\right|_{2}^{2} d s \leq \delta(\varepsilon, \lambda), \varepsilon, \lambda>0 .
$$

Hence $X_{\varepsilon}(t) \rightarrow X$ in $C\left(\left[0, T^{*}\right] ; H^{-1}(\mathcal{O})\right) \cap L^{2}\left(0, T ; L^{2}(\mathcal{O})\right)$ for $\varepsilon \rightarrow 0$. Moreover by (3.14) we see that

$$
\left|\frac{d X_{t}}{d t}\right|_{L^{1}(0, T ; \mathcal{Z})} \leq C, \forall \varepsilon>0
$$

and

$$
A_{\varepsilon}(t) X_{\varepsilon}(t) \rightarrow A(t) X(t) \text {, weakly in } \mathcal{Z}, \forall t \in\left[0, T^{*}\right] .
$$

Then letting $\varepsilon \rightarrow 0$ in (3.14) we get (3.13).

We shall now use the previous lemma to study existence and uniqueness of a solution for the equation

$$
\begin{cases}\frac{\partial \widetilde{X}}{\partial t}-\Delta \Psi(\widetilde{X})+\operatorname{div}\left(\widetilde{Y}_{X}(t) \eta\left(\gamma^{-1}(\widetilde{X})\right)\right)=F, & \text { in }\left(0, T^{*}\right) \times \mathcal{O}, \\ \widetilde{X}(0, \xi)=X_{0}(\xi), & \text { in } \mathcal{O}, \\ \widetilde{X}(t, \xi)=0, & \text { on }\left(0, T^{*}\right) \times \partial \mathcal{O} .\end{cases}
$$

By Lemma 9 we have the following result. 
Theorem 10 For $X_{0} \in L^{2}(\mathcal{O}), \widetilde{Y} \in L^{2}\left(0, T^{*} ;\left(L^{\infty}(\mathcal{O})\right)^{3}\right)$ and $F \in L^{2}\left(\left(0, T^{*}\right) \times \mathcal{O}\right)$ equation (3.15) has a unique solution

$$
\widetilde{X} \in W^{1,1}\left(\left[0, T^{*}\right], \mathcal{Z}\right) \cap L^{2}\left(\left[0, T^{*}\right] ; L^{2}(\mathcal{O})\right) \cap C\left(\left[0, T^{*}\right] ; H^{-1}(\mathcal{O})\right) .
$$

Moreover the map $\widetilde{Y} \rightarrow \widetilde{X}$ is continuous from $L^{2}\left(0, T^{*} ; H\right)$ to $L^{2}\left(\left(0, T^{*}\right) \times \mathcal{O}\right)$.

We can prove now the main result of this section.

Theorem 11 Let $X_{0} \in L^{2}(\mathcal{O})$ and $Y_{0} \in V$. Then, for $T^{*}$ sufficiently small, the system (3.3) has a solution

$$
Y \in W^{1,2}\left(\left[0, T^{*}\right] ; H\right) \cap L^{2}\left(0, T^{*} ;\left(H^{2}(\mathcal{O}) \cap H_{0}^{1}(\mathcal{O})\right)^{3}\right) \cap C\left(\left[0, T^{*}\right] ; V\right)
$$

and

$$
X \in C\left(\left[0, T^{*}\right], H_{0}^{1}(\mathcal{O})\right) \cap L^{2}\left(\left(0, T^{*}\right) \times H_{0}^{1}(\mathcal{O})\right) \cap W^{1,1}\left(\left[0, T^{*}\right] ; H^{-1}(\mathcal{O})\right) .
$$

Proof. We define the set

$$
\mathcal{M}=\left\{X \in L^{2}\left(\left(0, T^{*}\right) \times \mathcal{O}\right) ;|X|_{L^{2}\left(\left(0, T^{*}\right) \times \mathcal{O}\right)} \leq M\right\}
$$

and consider the operator

$$
\Gamma: \mathcal{M} \rightarrow \mathcal{M}
$$

which associates to each function $X \in L^{2}\left(\left(0, T^{*}\right) \times \mathcal{O}\right)$ the solution $\widetilde{X}$ to the equation

$$
\begin{cases}\frac{\partial \widetilde{X}}{\partial t}-\Delta \Psi(\widetilde{X})+\operatorname{div}\left(\widetilde{Y}_{X}(t) \eta\left(\gamma^{-1}(\tilde{X})\right)\right)=F, & \text { in }\left(0, T^{*}\right) \times \mathcal{O} \\ \widetilde{X}(0, \xi)=X_{0}(\xi), & \text { in } \mathcal{O}, \\ \widetilde{X}(t, \xi)=0, & \text { on }\left(0, T^{*}\right) \times \partial \mathcal{O}\end{cases}
$$

where $\widetilde{Y}_{X}(t)$ is the solution of the Navier-Stokes equation

$$
\left\{\begin{array}{l}
\frac{d \widetilde{Y}}{d t}+\nu \widetilde{A}(\widetilde{Y})+B(\widetilde{Y})-P_{X(t)}(\widetilde{Y})=G(X), \quad\left(0, T^{*}\right) \\
Y(0)=Y_{0}
\end{array}\right.
$$

We can write then

$$
\Gamma(X)=\tilde{X} .
$$

We are going to apply the Schauder fixed point theorem, and to this purpose we need to show that $\Gamma(\mathcal{M}) \subset \mathcal{M}, \Gamma$ is continuous on $L^{2}\left(\left(0, T^{*}\right) \times \mathcal{O}\right)$, and that $\Gamma(\mathcal{M})$ is relatively compact in $\mathcal{M}$. 
We shall show first that $\Gamma(\mathcal{M}) \subset \mathcal{M}$. Let $X \in \mathcal{M}$. We multiply the equation

$$
\frac{\partial \widetilde{X}}{\partial t}-\Delta \Psi(\widetilde{X})+\operatorname{div}\left(\widetilde{Y}_{X}(t) \eta\left(\gamma^{-1}(\widetilde{X})\right)\right)=F,
$$

scalarly in $L^{2}(\mathcal{O})$ by $\Psi(\tilde{X})$ and get

$$
\begin{aligned}
& \int_{\mathcal{O}} j(\widetilde{X}(t, \xi)) d \xi+\frac{1}{2} \int_{0}^{t}|\nabla \Psi(\widetilde{X})|_{2}^{2} d s \\
\leq & \int_{\mathcal{O}} j\left(X_{0}\right) d \xi+C \int_{0}^{t} \int_{\mathcal{O}}\left|\widetilde{Y}_{X}\right|_{\mathbb{R}^{3}}^{2}|\widetilde{X}|^{2} d \xi d s+\int_{0}^{t} \int_{\mathcal{O}} F \Psi(\widetilde{X}) d \xi d s \\
\leq & \int_{\mathcal{O}} j\left(X_{0}\right) d \xi+C \int_{0}^{t}\left|\widetilde{Y}_{X}\right|_{\left(L^{4}(\mathcal{O})\right)^{3}}^{2}|\widetilde{X}|_{L^{4}(\mathcal{O})}^{2} d s+C|F|_{L^{2}\left(\left(0, T^{*}\right) \times \mathcal{O}\right)}^{2} \\
\leq & \int_{\mathcal{O}} j\left(X_{0}\right) d \xi+C\left|\widetilde{Y}_{X}\right|_{L^{\infty}\left(0, T^{*} ; V\right)}^{2} \int_{0}^{t} \int_{\mathcal{O}}|\nabla \Psi(\widetilde{X})|^{2} d \xi d s+C|F|_{L^{2}\left(\left(0, T^{*}\right) \times \mathcal{O}\right)}^{2}
\end{aligned}
$$

where $j(r)=\int_{0}^{r} \Psi(s) d s$. (Here we have used Sobolev embedding theorem $\left(H_{0}^{1}(\mathcal{O}) \subset L^{4}(\mathcal{O})\right)$ and the fact that $\Psi^{\prime}(v)>\psi_{0}, \forall v$.

This leads to

$$
\begin{aligned}
& |\widetilde{X}(t)|_{2}^{2}+\int_{0}^{t}|\widetilde{X}|_{H_{0}^{1}(\mathcal{O})}^{2} d s \\
\leq & C\left(\left|X_{0}\right|_{2}^{2}+\left|\widetilde{Y}_{X}\right|_{L^{\infty}\left(0, T^{*} ; V\right)}^{2}+|F|_{L^{2}\left(\left(0, T^{*}\right) \times \mathcal{O}\right)}^{2}\right) .
\end{aligned}
$$

Taking into account that

$$
\left|\widetilde{Y}_{X}\right|_{L^{\infty}\left(0, T^{*} ; V\right)} \leq C L\left(\left|Y_{0}\right|_{V}^{2}+\int_{0}^{T^{*}}|\widetilde{X}(t)|_{2}^{2} d t\right) .
$$

We see by the above inequality that

$$
\begin{aligned}
& |\widetilde{X}(t)|_{L^{\infty}\left(0, T^{*} ; L^{2}(\mathcal{O})\right)}^{2} \\
\leq & C\left(\left|X_{0}\right|_{2}^{2}+|F|_{L^{2}\left(\left(0, T^{*}\right) \times \mathcal{O}\right)}^{2}+L\left|Y_{0}\right|_{V}^{2}+L T^{*} M^{2}\right) \\
\leq & M^{2}
\end{aligned}
$$

if $L T^{*} \leq \frac{1}{2}$ and $M$ is sufficiently large.

Hence $X \in \mathcal{M}$ if $M$ is chosen as above and $\left|Y_{0}\right|_{V}+\frac{1}{\Psi_{0}}$ is small enough, which means that $\Gamma(\mathcal{M}) \subset \mathcal{M}$.

The continuity of $\Gamma$ follows by the continuity of the maps $X \rightarrow Y$ in Theorem 7 and of $Y \rightarrow \widetilde{X}$ from Theorem 10.

We note that by (3.17) it follows also that

$$
|\widetilde{X}|_{L^{2}\left(0, T^{*} ; H_{0}^{1}(\mathcal{O})\right)}+\left|\frac{\partial}{\partial t} \widetilde{X}\right|_{L^{1}\left(0, T^{*} ; H^{-1}(\mathcal{O})\right)} \leq C_{1}
$$


We have also by (3.16) that

$$
\int_{0}^{t}|\Delta \Psi(\tilde{X})|_{-1}^{2} d s=\int_{0}^{t}|\nabla \Psi(\tilde{X})|_{2}^{2} d s \leq C_{2},
$$

by the previous estimate, and

$$
\int_{0}^{t}\left|\operatorname{div}\left(\widetilde{Y}_{X}(X) \eta\left(\gamma^{-1}(\widetilde{X})\right)\right)\right|_{-1}^{2} d s \leq C_{3} .
$$

We obtain that

$$
\left|\frac{\partial}{\partial t} \widetilde{X}\right|_{L^{1}\left(0, T^{*} ; H^{-1}(\mathcal{O})\right)} \leq C_{4} .
$$

Finally, by using the Aubin-Lions-Simon theorem (see [14]), [22]) it follows that $\Gamma(\mathcal{M})$ is compact in $L^{2}\left(\left(0, T^{*}\right) \times \mathcal{O}\right)$ as claimed.

We can now complete the proof of this result by applying the Schauder theorem and getting that the operator $\Gamma$ has a fixed point.

\section{Numerical illustration}

The phase-change problem studied in this paper is encountered in numerous practical applications, encompassing thermal energy storage (phase-change materials) and geophysical problems (Earth's mantle formation, lava lakes or magma chambers). For these applications, the classical Stefan model is not accurate, since temperature gradients induce buoyancy forces in the liquid (melted) phase generating a significant convective flow. Consequently, the mathematical modelling of the enthalpy equation has to include, as in our study, a velocity field in the liquid phase coming from a realistic fluid model. We used an adaptive finite-element method to solve the enthalpy equation (1.2), coupled with two different models for the liquid velocity $Y$ :

a) the complete Navier-Stokes system of equations (3.3) with Boussinesq approximation $f_{B}(\theta)=C \theta$ for thermal (buoyancy) effects and a Carman-Kozeny-type penalty term $g$ (3.4) to bring the velocity to zero in the solid phase;

b) a simplified flow model using a steady Darcy-Brinkman-type equation (as in porous media), i.e. we ignored in (3.3) the convective term $(Y \cdot \nabla) Y$ and replaced $d Y / d t$ by $Y$.

The numerical method is based on Lagrange finite-elements with dynamic mesh adaptivity. It was adapted from recent numerical studies of complex phasechange systems $[12,19]$. Model equations were discretized using Galerkin triangular finite elements, with Taylor-Hood elements for the flow (quadratic $\mathrm{P}_{2}$ for the velocity and piecewise linear $\mathrm{P}_{1}$ for the pressure) and $\mathrm{P}_{2}$ for enthalpy and temperature. The coupled system of equations was integrated in time using a fully-implicit backward Euler scheme. The resulting discrete non-linear equations was efficiently solved using a Newton algorithm. Consequently, we used 
a regularized Heaviside function $\tilde{H}$ (3.1) in the definition of the enthalpy (see Section 3).

An illustration of the numerical results is offered in Figure 1. We simulated a well-established numerical benchmark for phase-change problems, based on the experiments of [16]. It consists of a differentially heated square cavity, filled with octadecane paraffin. The material is initially solid and melts progressively starting from the left boundary, maintained at a hot temperature. The right boundary is also isothermal, with cold temperature. Horizontal boundaries are adiabatic. The physical (non-dimensional) parameters of the problem are the Rayleigh $(R a)$, Reynolds $(R e)$, Prandtl $(P r)$ and Stefan (Ste) numbers $R a=3.27 \cdot 10^{5}, R e=1, \operatorname{Pr}=56.2$ and $S t e=0.045$. The Boussinesq force then takes the form $f_{B}(\theta)=\frac{R a}{P r R e^{2}} \theta$ and the latent heat is $l=1 /$ Ste.

We plot in Figure 1 contours of the temperature field (left column) and streamlines in the liquid fraction (right column) for a given time instant $(t=$ 78.7) corresponding to the experimental measurement. The position of the liquid-solid interface (solid blue line) is compared to that reported in experiments (red dashed line). Good agreement with experimental results is obtained when the enthalpy equation is coupled with the Navier-Stokes equation for the liquid (Figure 1a). The same good agreement is obtained when the DarcyBrinkman model is used for the liquid (Figure 1b). For reference, we also simulated the classical Stefan problem, by decoupling the enthalpy equation from the Navier-Stokes model for the velocity (i.e. the div term was cancelled in Eq. (3.2)). As expected, ignoring the convection in the enthalpy equation results in a very poor approximation of the position of the liquid-solid interface (Figure $1 c)$.

The model studied in Section 3, coupling the enthalpy equation to the Navier-Stokes equation for the velocity, is the most complete existing model

phase-change systems with convection. It thus applies with good results to a wide range of applications [18]. The good results obtained using the DarcyBrinkman-type equation for the velocity are new and not reported elsewhere. This model was inspired by the present theoretical developments in Section 2. Its physical and numerical analysis opens new research paths in this area.

\section{Acknowledgements}

I.C. and I.D. acknowledge financial support from the French ANR grant ANR18-CE46-0013 QUTE-HPC and the European Union with the European regional development fund (ERDF, HN0002137 and 18P03390/18E01750/18P02733) and by the Normandie Regional Council (via the M2NUM and M2SiNum projects). V.B. acknowledges the hospitality of Laboratoire de mathématiques Raphaël Salem where this work was initiated in January 2020. 
a)
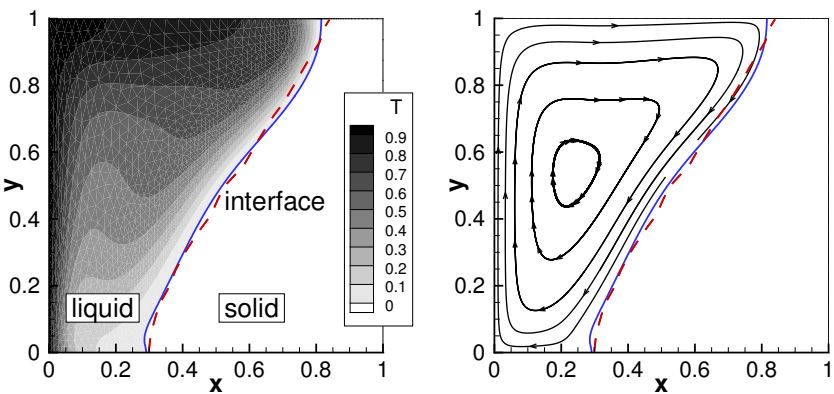

b)
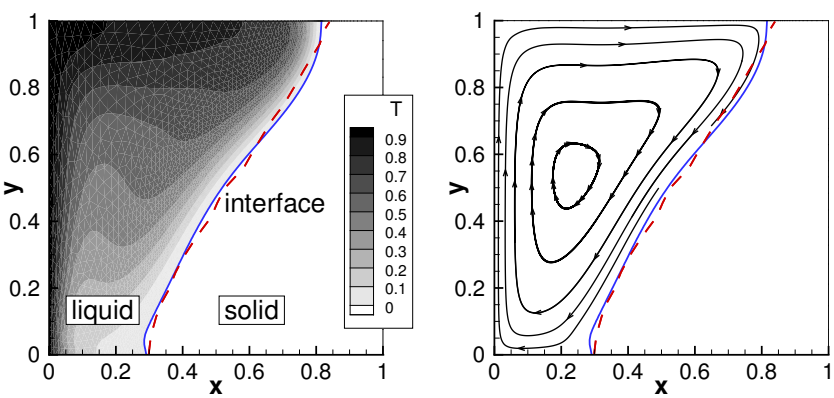

c)
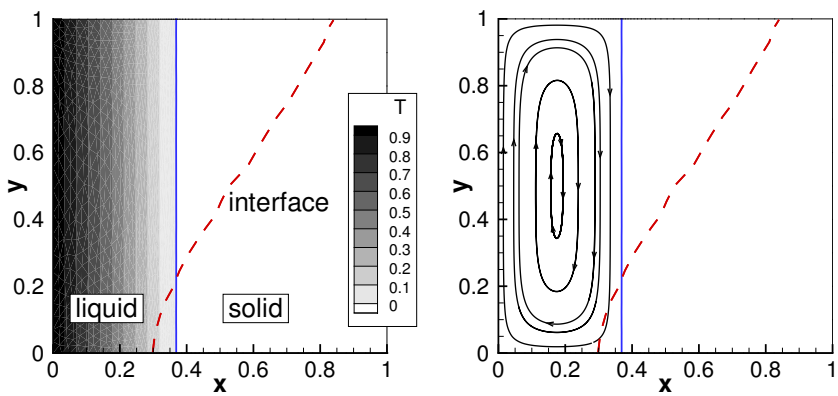

Figure 1: Numerical simulation of the melting of a phase change material (octadecane paraffin). The material is initially solid and melts progressively starting from the left boundary. Snapshots at the same time instant corresponding to experiments by [16]. Contours of the temperature field (left column) and streamlines (right column) in the liquid fraction. Liquid-solid interface extracted from simulations (solid blue line) and experiments (dashed red line). Models used for simulations: enthalpy equation coupled with the Navier-Stokes equation (a) or Darcy-Brinkmann-type equation (b) for the velocity. The classical Stefan model (c) is simulated for reference (the velocity and enthalpy equations are decoupled). 


\section{References}

[1] G. Amiez, P.-A. Gremaud, Error estimates for Euler forward scheme related to two-phase Stefan problems, ESAIM: Mathematical Modelling and Numerical Analysis, Volume 26, Issue 2, 365-383 (1992)

[2] V. Barbu, Nonlinear Differential Equations of Monotone Types in Banach Spaces, Springer, New York (2010)

[3] V. Barbu, Analysis and Control of Nonlinear Infinite Dimensional Systems, Volume 190, 1st Edition, Academic Press (1992)

[4] V. Barbu, M. Röckner, From nonlinear Fokker-Planck equations to solutions of distribution dependent SDE, arXiv:1808.10706V2, to appear in Ann. Prob. (2020)

[5] V. Barbu, M. Röckner, Solutions for nonlinear Fokker-Planck equations with measures as initial data and McKean-Vlasov equations, arXiv:2005.02311, submitted

[6] V. Barbu, M. Röckner, The evolution to equilibrium of solutions to nonlinear Fokker-Planck equation, arXiv:1904.08291, submitted

[7] D. Blanchard, A. Porretta, Stefan problems with nonlinear diffusion and convection, Journal of Differential Equations, Volume 210, Issue 2, 383-428 (2005)

[8] M. Boukrouche, G. Lukaszewicz, The Stationary Stefan Problem with Convection Governed by a Non-linear Darcy's Law, Math. Meth. Appl. Sci., 22, 563-585 (1999)

[9] H. Brezis, Functional Analysis, Sobolev Spaces and Partial Differential Equations, Springer (2011)

[10] M.G. Crandall, Generation of semigroups of nonlinear transformations in general Banach spaces, Amer. J. Math., 93, 265-298 (1971)

[11] C. M. Dafermos and M. Slemrod, Asymptotic behavior of nonlinear contraction semigroups, J. Functional Analysis 13, 97-106 (1973)

[12] I. Danaila, R. Moglan, F. Hecht, and S. Le Masson, A Newton method with adaptive finite elements for solving phase-change problems with natural convection, J. Comput. Physics, 274, 826-840 (2014)

[13] C.M. Elliot, J.R. Ockendon, Weak and Variational Methods for Moving Boundary Problems, Pitman Research Notes in Mathematics 59, Boston. London. Melbourne (1982)

[14] J.-L. Lions, Quelques méthodes de résolution des problèmes aux limites non linéaires, Dunod (2002) 
[15] S.L. Mitchell, M. Vynnycky, On the numerical solution of two-phase Stefan problems with heat-flux boundary conditions, Journal of Computational and Applied Mathematics, Volume 264 (2014)

[16] M. Okada, Analysis of heat transfer during melting from a vertical wall, International Journal of Heat and Mass Transfer, 27, 2057-2066 (1984)

[17] A. Pazy, The Lyapunov method for semigroups of nonlinear contractions in Banach spaces, Journal d'Analyse Mathématique 40(1), 239-262 (1981)

[18] A. Rakotondrandisa, I. Danaila, and L. Danaila, Numerical modelling of a melting-solidification cycle of a phase-change material with complete or partial melting, Int. J. of Heat and Fluid Flow, 76, 57-71 (2019)

[19] A. Rakotondrandisa, G. Sadaka, and I. Danaila, A finite-element toolbox for the simulation of solid-liquid phase-change systems with natural convection, Computer Physics Communications, to appear:doi 10.1016/j.cpc.2020.107188 (2020)

[20] T. Roubicek, The Stefan problem in heterogeneous media, Annales de l'Institut Henri Poincaré (C) Non Linear Analysis 6(6) (1989)

[21] L.I. Rubinstein, The Stefan Problem, Translations of Mathematical Monographs, Vol. 27, American Mathematical Society (1971)

[22] Simon, J. Compact sets in the space $\operatorname{Lp}(\mathrm{O}, \mathrm{T} ; \mathrm{B})$. Annali di Matematica pura ed applicata 146, 65-96 (1986)

[23] D. Tarzia, A bibliography on moving-free boundary problems for the heatdiffusion equation. The Stefan and related problems, M.A.T. Seria A: Mathematical Conferences, Seminar and Papers, Vol. 2 (2000) 